\title{
Guided wave mode selection for inhomogeneous elastic waveguides using frequency domain finite element approach
}

\author{
Vamshi Krishna Chillara ${ }^{\mathrm{a}, *}$, Baiyang Ren ${ }^{\mathrm{a}}$, Cliff J Lissenden ${ }^{\mathrm{a}}$ \\ ${ }^{a}$ Department of Engineering Science and Mechanics, The Pennsylvania State University, \\ $16802 P A$
}

\section{Abstract}

This article describes the use of the frequency domain finite element (FDFE) technique for guided wave mode selection in inhomogeneous waveguides. Problems with Rayleigh-Lamb and Shear-Horizontal mode excitation in isotropic homogeneous plates are first studied to demonstrate the application of the approach. Then, two specific cases of inhomogeneous waveguides are studied using FDFE. Finally, an example of guided wave mode selection for inspecting disbonds in composites is presented. Identification of sensitive and insensitive modes for defect inspection is demonstrated. As the discretization parameters affect the accuracy of the results obtained from FDFE, effect of spatial discretization and the length of the domain used for the spatial fast

\footnotetext{
${ }^{*}$ Corresponding author

Email address: vkc5017@psu.edu; Ph:814-954-2291 (Vamshi Krishna Chillara )
} 
Fourier transform are studied. Some recommendations with regard to the choice of the above parameters are provided.

Keywords: Ultrasonics; Guided waves; Frequency-domain; Finite elements;

\section{Introduction}

Ultrasonic guided waves are widely used as a tool for a variety of problems in nondestructive evaluation (NDE) and structural health monitoring (SHM). As opposed to bulk waves which travel in unbounded media, guided waves require boundaries to guide the energy in the structure. Also, there exists multiple guided wave modes in the structure owing to the nature of the eigenvalue problem associated with the structure. Hence, guided wave mode-selection [1] is the key to identify specific kinds of defects. For this, it is necessary to have a thorough understanding of the characteristics of wave propagation in waveguides. Two essential features that characterize the wave propagation in a homogeneous waveguide, where there are no changes (material or geometric) along the length of the waveguide, are dispersion curves and wavestructures [2].

While dispersion curves depict the frequency-wavenumber $(\omega, \mathrm{k})$ combinations at which propagating guided wave modes exist in the structure, wavestructures describe the through-thickness displacement/stress profiles 
for the guided wave modes. Both dispersion curves and wavestructures guide the choice of a mode and frequency for a particular application. While analytical solutions to obtain dispersion curves and wavestructures are available for simple geometries like those of homogeneous plates and pipes [2, 3], numerical methods are the only resort for more complicated geometries. Several approaches have been used in the past to address the above issue [4]. The finite difference approach and the finite element method [5](FEM) are by far the most commonly used numerical techniques to study wave propagation in inhomogeneous waveguides. Even though time-domain transient dynamic analysis using FEM has the capability to simulate wave propagation in structures of large sizes and complex shapes, it requires a lot of computational effort in most cases. Hence, other approaches such as the semi-analytical finite element method (SAFE) [6], which is a hybrid approach combining analytical and finite element methods, are being used to study homogeneous waveguides. While it is possible to obtain dispersion curves and wavestructures for waveguides which are homogeneous in the propagation direction using SAFE, inhomogeneous waveguides, where the material or geometry changes along the length of the waveguide, call for a different approach. Of particular interest are functionally graded materials, inhomogeneous periodic 
waveguides and waveguide transitions [7].

For example, the problem of guided wave mode conversion across a discrete waveguide transition [7] is studied using SAFE and the normal mode expansion technique. The approach was to use solutions from SAFE to model the wave field in the homogeneous regions before and after the transition and then use the appropriate boundary conditions at the transition to determine the modal content in the respective wave fields. It is noted that such a transition is a rather simple inhomogeneity and such good workable solutions are not known for more complex inhomogeneous waveguides, impairing the efficiency of NDE/SHM in inspecting them. This article demonstrates the use of a Frequency Domain Finite Element (FDFE) approach for guided wave mode selection for NDE/SHM of inhomogeneous waveguides. The FDFE approach has previously been used to study the scattering from defects and reflection and transmission of waves across interfaces [8, 9, 10, and waveguide transitions [11]. The corresponding results are compared with those from time-domain Finite Element approach and are found to be in good agreement. To the best of our knowledge, the above approach has not been employed for guided wave ultrasonic inspection, so we address this practical issue here. The advantages of FDFE relative to time-domain analysis 
include: computational efficiency, it permits study of mode conversion, it gives phase velocities as opposed to group velocity, and it enables an efficient method to select modes sensitive to damage.

The content of this article is organized as follows. Section 2 presents the background, theory and formulation of the elastic boundary value problem in the frequency domain. Then section 3 demonstrates the approach for an isotropic homogeneous plate where the phase velocities and wavestructures of Rayleigh-Lamb (RL) and Shear-Horizontal modes (SH) are recovered. Next, section 4 presents the results obtained from applying FDFE for two cases of inhomogeneous waveguides. There are many types of inhomogeneous waveguides that are of practical importance for NDE and SHM. Damage localization is one good example. The change in energy transmitted through the damaged region is necessary for through-transmission methods and the wave energy reflected by the damaged region is required for pulse-echo methods. The idea of mode selection using FDFE is outlined and the special case of a disbond in an adhesively bonded joint between composite laminates is presented as an example. The effect of parameters such as discretization on the solution of FDFE is presented in section 5 and the conclusions are drawn in section 6 . 


\section{Frequency Domain Finite Element Approach (FDFE)}

The governing equations for a linear elastic waveguide [12] are given by

$$
\begin{aligned}
\nabla \cdot \sigma & =\rho \ddot{u} \quad(\text { Balance of linear momentum) } \\
\sigma & =C \epsilon(\text { Constitutive relation) } \\
\epsilon & =\frac{1}{2}\left(\nabla u+(\nabla u)^{T}\right) \quad \text { (strain-displacement relation) }
\end{aligned}
$$

where $\sigma$ denotes Cauchy stress, $C$ represents the tensor of elastic moduli, $\epsilon$ is the strain tensor, $u$ denotes the displacement field and $\rho$ denotes the mass-density of the material. The frequency domain finite element approach attempts to solve Eqns(1-3) in the frequency domain. Solution in the frequency domain gives the characteristics of a system in terms of the frequency response function/modal response function. Just as one can determine a frequency response function for a particle system, one could extend a similar concept to characterize the structural behavior of the waveguide.

Consider the forced vibration of a particle attached to a spring as shown in Figure 1. The frequency response function can be written as $H(\omega)=1 /(\kappa-$ $\left.m \omega^{2}\right)$, where $\kappa, m$, and $\omega$ are stiffness, mass, and frequency respectively. The natural frequency of the system is obtained by setting $\kappa-m \omega^{2}=0$. Likewise, 
a continuum can be thought of as an infinite system of particles and hence it has infinite natural frequencies. Similarly, a waveguide could be thought of as a vibrating continuum whose response can be captured in a modal response function/transfer function, which could be described as $H(\omega, k)$ where $\omega$ denotes the frequency and $k$ the wavenumber. The propagating wave modes can be identified as zeros of the transfer function $H(\omega, k)$. For example, $H(\omega, k)$ for the wave propagation in an isotropic, homogeneous, traction free plate [2] is given by $H(\omega, k)=\left(D_{s}(\omega, k) D_{a}(\omega, k) D_{S H}(\omega, k)\right)^{-1}$, where

$$
\begin{aligned}
D_{s}(\omega, k) & =\frac{\tan (q h)}{\tan (p h)}+\frac{4 k^{2} p q}{\left(q^{2}-k^{2}\right)^{2}} \quad \text { Symmetric RL modes } \\
D_{a}(\omega, k) & =\frac{\tan (q h)}{\tan (p h)}+\frac{\left(q^{2}-k^{2}\right)^{2}}{4 k^{2} p q} \quad \text { Antisymmetric RL modes } \\
D_{S H}(\omega, k) & =q h-\frac{n \pi}{2} ; n=1,2, \cdots ; \quad \text { SH modes }
\end{aligned}
$$

where $p=\sqrt{\left(\frac{\omega^{2}}{c^{2}}-k^{2}\right)}$ and $q=\sqrt{\left(\frac{\omega^{2}}{c t^{2}}-k^{2}\right)}$, with $c_{l}$ and $c_{t}$ representing the longitudinal and transverse wave speeds in the material.

The function $H(\omega, k)$ could be explicitly determined either analytically or numerically as above in very few cases. For the case of inhomogeneous waveguides or waveguides with complex geometries, it may not be possible 
for a single wavenumber $k$ to characterize the displacement field in the entire waveguide at a given frequency $\omega$ owing to the inhomogeneous nature of the waveguide. Hence it is an onerous task to identify a particular mode suitable for a given NDE/SHM application. One alternative approach is to study the wave propagation for various excitations (boundary conditions) using time-domain FEM techniques, use the time-domain data to determine the displacement field, and then apply a 2DFFT (spatio-temporal fast Fourier transform) to determine the frequency-wavenumber content in the displacement field.

The other approach, i.e., FDFE, attempts to solve the problem for time harmonic excitations; i.e., in the frequency domain. It assumes a time harmonic nature of displacement, stress and strain fields and solves Eqns(1-3) at each frequency. To be specific, we assume $u=u(\hat{x}) e^{i \omega t}$ and $\sigma=\sigma(\hat{x}) e^{i \omega t}$ where $\hat{x}$ denotes the position of the material point in Cartesian space $(x, y, z)$. The balance of linear momentum under the above assumptions simply becomes

$$
\nabla \cdot \sigma(\hat{x})+\rho \omega^{2} u(\hat{x})=0
$$

Note that time-derivatives are eliminated with the time-harmonic assumption. Importantly for the problems at hand, any inhomogenieties in 
the material properties or the geometry can be incorporated in the finite element discretization. Equation (7) is solved using FEM to obtain $u(\hat{x})$ at each frequency $\omega$. The advantage of this approach is that the computational cost is considerably reduced because we end up solving a pseudo-static problem that requires no time-stepping as in the time-domain finite element method. Furthermore, the source-influence [2] leading to multimode excitation in time-domain finite element analyses is minimized due to a single frequency component in the analysis.

\section{FDFE - Isotropic homogeneous elastic plate}

All the results presented in this article are obtained using the frequency domain solver in the solid mechanics module of COMSOL Multiphysics [13]. Through the following examples we demonstrate that FDFE is capable of accurately predicting the displacement field. We consider the excitation of both the RL and SH modes in a traction free plate. The schematic of the model used for simulations is shown in Figure 2. The length of the waveguide is $100 \mathrm{~mm}$ and its thickness is $1 \mathrm{~mm}$. A Perfectly Matched Layer (PML) of length $50 \mathrm{~mm}$ and thickness $1 \mathrm{~mm}$ is used at the end of the waveguide to absorb reflections. Also, fixed boundary conditions are enforced on the right 
extreme of PML. Quadrilateral elements with a maximum element size of $0.1 \mathrm{~mm}$ are used to discretize the domain i.e., both the waveguide and PML. This choice ensured ten elements through the thickness and 1000 elements along the length of the waveguide. The excitation is specified as displacement boundary conditions (BC's) at the left end of the plate. FDFE is applied to obtain the displacement field in the plate at different frequencies. Depending on the nature of excitation, different modes will be excited in the structure. For example, if we wish to excite symmetric RL modes in the structure we specify a symmetric through-thickness profile for the displacement BCs. We denote the $x, y$ and $z$ components of the displacement by $\mathrm{U}_{1}, \mathrm{U}_{2}$ and $\mathrm{U}_{3}$ respectively. In all the cases that follow, displacements $\mathrm{U}_{1}$ or $\mathrm{U}_{3}$ are specified depending on whether we want to excite $\mathrm{RL}$ or $\mathrm{SH}$ modes and the displacement component $\mathrm{U}_{2}$ is unconstrained. We also illustrate the procedure for computing the phase velocity of the mode by using the spatial Fourier transform to obtain the wavenumber. Before demonstrating the method, we show the dispersion curves (Figure 3) for an aluminum plate $\left(\mathrm{E}=70 \mathrm{GPa} ; \nu=0.33 ; \rho=2700 \mathrm{~kg} / \mathrm{m}^{3}\right)$ for which the results in this section are obtained. Only the three fundamental modes $\left(\mathrm{S}_{0}, \mathrm{~A}_{0}\right.$, and $\left.\mathrm{SH}_{0}\right)$ exist for low frequencies. Aside from the $\mathrm{SH}_{0}$ mode, all modes are dispersive. It is the 
in-plane through-thickness displacement profile $\left(\mathrm{U}_{1}\right)$ that indicates whether an RL mode is symmetric or antisymmetric.

\subsection{Shear horizontal mode excitation}

In this section we present the FDFE results obtained for three different SH mode excitations: uniform $\mathrm{U}_{3}$ displacement at $0.5 \mathrm{MHz}$ and linear antisymmetric $\mathrm{U}_{3}$ displacement at $2 \mathrm{MHz}$ and $0.5 \mathrm{MHz}$. For homogeneous isotropic materials the SH and RL modes are decoupled. Thus, in this section only the $\mathrm{U}_{3}$ displacement component is considered.

\section{Displacement BC-Uniform $\mathrm{U}_{3}$ through the thickness of the plate}

A displacement $\mathrm{BC}$ of $\mathrm{U}_{3}=1$ is applied to the left end of the plate. The nodal displacements are then computed for $0.5 \mathrm{MHz}$ using FDFE. Then a spatial FFT is computed from the displacement field to determine the wavenumber content. The frequency-wavenumber $(\omega, k)$ content provides the desired wave propagation features. Results are presented in Figure 4 , which shows:

(a) a snapshot of the $\mathrm{U}_{3}$ displacement component throughout the domain indicated as an intensity map, 
(b) a plot of the $\mathrm{U}_{3}$ displacement profile through the thickness of the plate (commonly known as the wavestructure); this profile is plotted at $x=$ $50 \mathrm{~mm}$ from the left end of the plate,

(c) a plot of the $\mathrm{U}_{3}$ displacement component along $x$ on the top-surface of the plate i.e., $y=1 \mathrm{~mm}$ and

(d) the wavenumber spectrum obtained from the spatial FFT of $\mathrm{U}_{3}$ along $x$ on the top surface of the plate.

Note that the plots above show only the displacement in the waveguide but not in the PML. Similar plots are given in the subsequent examples. The wavestructure shown in Figure $4 \mathrm{~b}$ is that of the $\mathrm{SH}_{0}$ mode. The wavenumber obtained using the spatial FFT (Figure 4d) is $k=1004 m^{-1}$, which enables computation of the phase velocity $c_{p}=\frac{\omega}{k}=3.1 \mathrm{~mm} / \mu \mathrm{s}$. The phase velocity of the $\mathrm{SH}_{0}$ mode in Figure 3 is $3.10 \mathrm{~mm} / \mu \mathrm{s}$. The wave field features shown in Figure $4 \mathrm{~b}$ and the excellent agreement of phase velocities indicate that the $\mathrm{SH}_{0}$ mode is the one and only mode present - as it should be. 


\section{Displacement BC-Linear variation in $\mathrm{U}_{3}$ from -1 to 1 through the thickness of the plate}

Applying the linear antisymmetric displacement $\mathrm{BCs}$ for $\mathrm{U}_{3}$ at a frequency of $2 \mathrm{MHz}$ provides the results shown in Figure 5. These BCs excite the $\mathrm{SH}_{1}$ mode whose wavestructure is shown (Figure 5b) to compare well with that from the analytical solution. The wavenumber obtained using the spatial

Fourier transform (Figure 5d) is $k=2511 m^{-1}$. The computed phase velocity is $c_{p}=\frac{\omega}{k}=5 \mathrm{~mm} / \mu \mathrm{s}$, while the actual phase velocity from Figure 3 is 4.92 $\mathrm{mm} / \mu \mathrm{s}$.

When the same linear antisymmetric displacement BCs are applied at $0.5 \mathrm{MHz}$ the results in Figure 6 are obtained. Figure $6 \mathrm{~b}$ show that the disturbance dies out rapidly in the $x$ direction indicating the presence of an evanescent mode. Here, we are trying to excite an antisymmetric SH mode at $0.5 \mathrm{MHz}$ where, in fact, there is no propagating mode. This example was chosen to demonstrate that FDFE is capable of identifying the existence/nonexistence of modes for a particular boundary condition.

\subsection{Rayleigh-Lamb mode excitation}

In this section we present examples of RL mode excitation using FDFE. 


\section{Displacement BC-Uniform $\mathrm{U}_{1}$ through the thickness of the plate}

A uniform $\mathrm{U}_{1}=1$ displacement field is prescribed as the $\mathrm{BC}$ at the left end of the plate. FDFE is employed to obtain the displacement field at a $0.5 \mathrm{MHz}$ excitation. Figure $7 \mathrm{a}$ shows an intensity map of the displacement fields $\left(\mathrm{U}_{1}\right.$ and $\left.\mathrm{U}_{2}\right)$. The displacements (Figure $7 \mathrm{~b}$ ), wavestructure (Figure 7c) and the wavenumber content (Figure 7d) correspond to the S0 mode. Also note that the expected phase difference of $\pi / 2$ between the $\mathrm{U}_{1}$ and $\mathrm{U}_{2}$ displacements is evident in Figure $7 \mathrm{~b}$. The computed phase velocity from Figure $7 \mathrm{~d}$ is $c_{p}=\frac{\omega}{k}=5.6 \mathrm{~mm} / \mu \mathrm{s}$, while the actual phase velocity from Figure 3 is $5.35 \mathrm{~mm} / \mu \mathrm{s}$. The error in the phase velocity estimate is less than $5 \%$. It should be noted that the phase velocity is computed using the wavenumber corresponding to the peak value in spatial FFT (Figure 7d). For a more refined estimate one could take an average of the phase velocity estimate across the points in the wavenumber bandwidth of the spatial FFT.

\section{Displacement BC-Sinusoidal $\mathrm{U}_{1}$ through the thickness}

A displacement given by $\mathrm{U}_{1}(0, y)=\sin (2 \pi(2 y-1))$ is applied to the plate at the left end, where $y$ is in $\mathrm{mm}$. FDFE is employed to compute the displacements at frequencies of 0.5 and $2 \mathrm{MHz}$. Due to the antisymmetric nature of the displacement field through the thickness, it is expected that 
the outcome will be an antisymmetric mode.

We first discuss the results obtained at $0.5 \mathrm{MHz}$ shown in Figure 8, Figure 8a shows the displacement field indicating that it is an antisymmetric mode further confirmed by the wavestructure shown in Figure $8 \mathrm{c}$. The wavenumber obtained from Figure $8 \mathrm{~d}$ is $k=1695 \mathrm{~m}^{-1}$ which enables the computation of phase velocity as $c_{p}=\frac{\omega}{k}=1.9 \mathrm{~mm} / \mu \mathrm{s}$. This is in very good agreement with the actual value obtained from Figure 3 i.e., $1.87 \mathrm{~mm} / \mu \mathrm{s}$.

Next we discuss the results obtained for a $2 \mathrm{MHz}$ excitation. At $2 \mathrm{MHz}$ there are two antisymmetric modes; $A_{0}$ and $A_{1}$ as shown in Figure 3. The results shown in Figure 9 indicate that both modes are present. This is clearly evident in Figure $9 \mathrm{c}$ as there are two peaks in the FFT corresponding to the $\mathrm{A}_{0}$ and $\mathrm{A}_{1}$ modes. The computed phase velocities are $2.7 \mathrm{~mm} / \mu \mathrm{s}$ for the $\mathrm{A}_{0}$ mode and $9.1 \mathrm{~mm} / \mu \mathrm{s}$ for the $\mathrm{A}_{1}$ mode, which agree favorably with the actual phase velocities in Figure 3 of $2.66 \mathrm{~mm} / \mu \mathrm{s}$ and $8.8 \mathrm{~mm} / \mu \mathrm{s}$ respectively. This example demonstrates the capability of FDFE to predict the existence of multiple modes corresponding to the imposed boundary conditions. This is very important when studying applications involving waveguide transitions [1] where multiple mode conversions are common. 


\section{FDFE - Inhomogeneous waveguides}

In this section we present results for FDFE applied to inhomogeneous waveguides. First, two simple cases have been selected to demonstrate the applicability of FDFE:

1. Bi-material plate i.e., a plate composed of two homogeneous isotropic materials that are perfectly bonded together in the middle.

2. Plate with linearly graded isotropic properties (Young's modulus) along the wave propagation direction.

Then the approach is employed for guided wave mode selection for detecting disbonds in a quasi-isotropic composite plate.

\subsection{Bi-material plate}

\section{Displacement BC-Uniform $\mathrm{U}_{1}=1$ through the thickness of the plate}

The model used to simulate the bimaterial plate is similar to the one in Figure 2 except that the region to the left of the PML is composed of plates made of two materials each $100 \mathrm{~mm}$ in length. The material properties for the plates are chosen to be that of aluminum $(\mathrm{E}=70 \mathrm{GPa} ; \nu=0.33$; $\left.\rho=2700 \mathrm{~kg} / \mathrm{m}^{3}\right)$ and copper $\left(\mathrm{E}=110 \mathrm{GPa} ; \nu=0.35 ; \rho=8700 \mathrm{~kg} / \mathrm{m}^{3}\right)$ with aluminum on the left-half and copper on the right-half of the plate. 
Boundary conditions are imposed in order to excite the $\mathrm{S}_{0}$ mode at 0.5 $\mathrm{MHz}$ on the left side of the bi-material plate. The results are shown in Figure 10. The displacement components $\mathrm{U}_{1}$ and $\mathrm{U}_{2}$ plotted in Figure 10a indicates that the acoustic impedance mismatch at the sharp interface results in a reflection and reduction in energy transmitted across the interface. The wavenumber content shown in Figure $10 \mathrm{~b}$ was obtained by Fourier transform

of displacements on either side of the interface. Clearly, the wavenumber in aluminum is different than in copper, which is expected since the phase velocities of $\mathrm{S}_{0}$ mode $(0.5 \mathrm{MHz})$ in aluminum and copper are 5.34 and $3.76 \mathrm{~mm} / \mu \mathrm{s}$ respectively. The computed phase velocities are $5.27 \mathrm{~mm} / \mu \mathrm{s}$ and $3.8 \mathrm{~mm} / \mu \mathrm{s}$ respectively.

\subsection{Linear Variation of Young's modulus}

Here the Young's modulus of the plate is varied from left to right end of the plate linearly as $E(x)=70(1+10 \alpha x) \mathrm{GPa}$ where $x$ is in ' $\mathrm{m}$ ' and $\alpha$ is a parameter used to represent the functionally graded material.

\section{Displacement BC-Uniform $\mathrm{U}_{1}=1$ through the thickness of the plate}

Boundary conditions are imposed to excite the S0 mode at $0.5 \mathrm{MHz}$ on the left end of the linearly graded plate. The results obtained for the dis- 
placement field $\mathrm{U}_{1}$ are shown in Figure 11a. As can be seen, the amplitude decreases along the propagation length ( $x$ direction) due to the continuous change in material properties. Also, shown in Figure $11 \mathrm{~b}$ is the spatial FFT that indicates the peak wavenumber for both the cases is different.

\subsection{Mode selection in inhomogeneous waveguides using FDFE}

The goal of mode selection is to identify appropriate guided wave modes sensitive to a specific targeted type of defect. Here, we briefly describe how FDFE can be used for mode selection in inhomogeneous waveguides.

Presume that the guided wave modes are reasonably well known at the location where transducer is placed to excite the waves. These guided waves propagate along the structure and get transformed into different modes owing to the inhomogeneous nature of the waveguide and/or due to the presence of defects i.e., essentially due to the boundary/interface conditions. To identify a specific kind of defect, it is essential that we select that mode which is most affected by the targeted type of defect. In many cases, the above requirement can be stated in terms of the particle displacement, shear and normal stresses or some combination thereof at the location of the defect. If the waveguide is inhomogeneous, the characteristics of the wave-field at the location of the defect can be drastically different than what we excite 
initially. Hence, we need to study the response of the waveguide for different possible excitations and select that particular mode which ensures desired characteristics of particle displacements and stresses at the location of the defect of interest. This can be accomplished easily by using FDFE to:

- Study waveguide characteristics along the propagation length for different frequencies spanning all possible modes at potential transducer locations,

- Change boundary conditions or add damage at appropriate locations to assess their effects on waveguide characteristics,

- Obtain wavenumbers using spatial FFT, which define the guided wave modes $(\omega, k)$.

Here, we present an example where the above procedure is successfully employed to identify sensitive guided wave modes for inspection of disbond in adhesive joints between quasi-isotropic laminated composite plates. Experimental results confirming the efficiency of mode selection are presented elsewhere [14]. 


\subsection{FDFE-Disbond in adhesive joint of composite plate}

Two laminates, each of 16 layers, are adhesively bonded together to form the composite plate and a disbond is artificially introduced during the bonding. The schematic of the plate with the disbond is shown in Figure 12 and the other parameters describing the lay-up of the composite are listed in Table 1, 2 and 3. As we are interested in the guided wave modes sensitive to the disbond, it is essential to incorporate some kind of absorbing boundary conditions [8] to avoid reflections from the ends of the plate. Here, perfectly matched layers (PML) are used for the absorbing boundary conditions.

The dispersion curves for the pristine bonded composite plate are shown in Figure 13. Through-thickness displacement fields (wavestructures) obtained from SAFE are applied as boundary conditions on the vertical line marked in Figure 12 to excite appropriate modes. However, analysis is required to ascertain which mode/frequency combinations are sensitive to the prescribed defect. Therefore, the interaction of four different guided wave modes with the disbond are studied as demonstration; Mode $1(210 \mathrm{kHz})$, Mode $2(310 \mathrm{kHz})$, Mode $6(310 \mathrm{kHz})$ and Mode $7(540 \mathrm{kHz})$. Each of these points are indicated in red on the dispersion curves. A sample wavestructure obtained using SAFE is shown for Mode $6(310 \mathrm{kHz})$ in Figure 14 . The $\mathrm{U}_{1}$ 
and $\mathrm{U}_{2}$ displacement fields obtained from FDFE are presented in Figure 15 for each of the above modes. Clearly, the displacement field for Mode 1 (210 $\mathrm{kHz}$ ) shows no appreciable difference to the right of the disbond. Hence, it is termed as an "insensitive" mode. On the other hand, the other three modes show considerable differences in the displacement field before and after the disbond. In fact, they exhibit considerable level of mode conversion across the disbond region. Hence these are termed as "sensitive" modes and can be used to identify the disbond. It should be noted that the terms sensitive and insensitive are with reference to the inspection being conducted in a throughtransmission mode, where the transmitter is located before the disbond and the receiver is located after the disbond. We note that only a subtle change occurs in Figure 15c, which is quite difficult to see given the scale in the figure. However, mode conversion does occur as is clearly shown in [14]. We should also point out that the difference in the displacement fields shown in [14] and $15 \mathrm{c}$ is due to using traction rather than displacement boundary conditions. Figures 16 and 17 show the spatial FFTs of both $U_{1}$ and $U_{2}$ for the modes $1(210 \mathrm{kHz})$ and $2(310 \mathrm{kHz})$. Here "left-to-right" denotes the right-propagating wave and "right-to-left" denotes the left-propagating wave so that the right-to-left propagating wave before the disbond corresponds to 
a reflection from the disbond and the left-to-right propagating wave after the disbond corresponds to the transmitted wave across the disbond. The left-to-right and right-to-left propagating waves are distinguished by the sign of the wavenumber in the spatial FFT of the displacement field. Clearly, all the figures show no right-to-left propagating waves after the disbond; this is because the PML on the right side absorbs all the energy in the left-to-right propagating waves resulting in no reflections. Also, as observed in Figure 15a, the FFTs for the Mode $1(210 \mathrm{kHz})$ in Figure 16 show no appreciable difference before and after the disbond. However, there is a small reflected component of another mode $\left(k=20 m^{-1}\right)$ visible in the FFT of $\mathrm{U}_{1}$ before the disbond. In addition, there are side lobes present in the FFT due to the zero-padding used before taking the FFT. On the other hand, Figure 17 shows considerable differences in the FFTs for both $\mathrm{U}_{1}$ and $\mathrm{U}_{2}$ before and after the disbond. First, there is a reduction in the amplitude of the mode $\left(k=112.3 \mathrm{~m}^{-1}\right)$ after the disbond. Correspondingly, there is an increase in the amplitude of the other mode $\left(k=48.83 \mathrm{~m}^{-1}\right)$. Although not shown here, similar results were obtained for the other modes; Mode $6(310 \mathrm{kHz})$ and the Mode 7 (540 kHz). Experimental investigations [14] were carried out in the through-transmission mode with all the above modes and the three 
"sensitive" modes were found to detect the disbond while the "insensitive" mode was unable to identify it. This study demonstrates the efficacy and practicality of the FDFE for guided wave mode selection from an inspection standpoint.

\section{Factors affecting numerical accuracy of solutions}

Two factors worthy of further analysis because they affect the accuracy of the solution predicted by FDFE are:

1. Spatial discretization, and

2. Length of the structural domain over which the spatial FFT is taken.

We illustrate the effect of both on the solution by considering the RayleighLamb mode excitation in a homogeneous plate.

\subsection{Effect of spatial discretization - $A_{0}$ mode $(0.5 \mathrm{MHz})$}

Figure 18 shows the $\mathrm{U}_{1}$ displacement component plotted along the $100 \mathrm{~mm}$ length of the waveguide for different discretizations where the size of each element along $x$ direction is varied $(1,0.8,0.4,0.2$ and $0.1 \mathrm{~mm})$ while keeping the discretization through the thickness constant $(0.1 \mathrm{~mm}) . \quad \mathrm{A}_{0}$ mode $(0.5$ $\mathrm{MHz}$ ) is excited using appropriate boundary conditions. The wavelength for 
this mode and frequency combination is $\approx 3.8 \mathrm{~mm}$. The spatial discretization along the length corresponds to $\lambda / 3.8, \lambda / 4.75, \lambda / 9.5, \lambda / 19$, and $\lambda / 38$ respectively. Figure 18 indicates that the displacement field has essentially converged for $(\lambda / 19)$ even though $\lambda / 9.5$ does provide a reasonable approximation to the displacement field. This is inline with time-domain finite element recommendation for discretization i.e., between $\lambda / 10$ and $\lambda / 20$ for the maximum element-size.

\subsection{Effect of the waveguide length}

As the accuracy of the wavenumber $(k)$ is the key to identifying the guided wave mode in the structure, it is necessary to study the factors that affect its accuracy. Suppose that $\mathrm{N}$ elements having edge length $\mathrm{x}$ are used to discretize the domain, then the resolution of the wavenumber is given by $2 \pi /((N+1) x) \approx 2 \pi / L$. Therefore the resolution in the wavenumber domain is governed by the length $(\mathrm{L})$ of the domain used for the spatial FFT. To demonstrate this, FDFE is run for waveguides of different length by keeping the spatial discretization constant. The $\mathrm{S}_{0}$ mode is excited at $0.5 \mathrm{MHz}$ using appropriate boundary conditions. The normalized spatial FFTs for the $\mathrm{S}_{0}$ mode at $0.5 \mathrm{MHz}$ is shown in Figure 19 for waveguide lengths of 100, 80, 60 and $40 \mathrm{~mm}$. The element size is $\Delta x=0.1 \mathrm{~mm}$. The figure indicate that 
as the waveguide becomes longer, the wavenumber peak becomes narrower, providing better resolution. This is intuitive, and in agreement with signal processing knowledge that a longer window length for FFT provides higher resolution in the frequency.

\section{Conclusions}

The formulation of the elastic boundary value problem in the frequency domain was presented in this article. The resulting governing partial differential equations were solved using the frequency domain finite element (FDFE) approach in COMSOL. We demonstrated how this approach can be used for guided wave mode selection in inhomogeneous waveguides. Using specific examples of guided wave modes in homogeneous plates, we demonstrated the ability of FDFE to identify the appropriate guided wave modes depending on the boundary conditions. Use of a spatial FFT to extract the wavenumber information from the displacement field was presented. Phase velocities and wavestructures obtained from FDFE were compared to those obtained from SAFE and were found to be in good agreement. The approach was employed to study two specific cases of inhomogeneous waveguides, namely one with a sharp interface and the other a functionally graded plate with a continuous 
variation of Young's modulus along the length of the waveguide. The efficacy of the FDFE approach for guided wave mode selection was demonstrated by identifying sensitive and insensitive modes for detecting disbonds in the adhesive joint of a composite plate. Experimental results presented elsewhere [14 corroborate the predictions. The effect of spatial discretization and the length of the waveguide on the FFT and hence the accuracy of FDFE in identifying the guided wave modes was studied using waveguides of different length. It was concluded that the longer the length of the waveguide, the higher is the accuracy in wavenumber for a given spatial discretization. For a given waveguide length, the finer the spatial discretization, the higher is the accuracy of guided wave mode identification.

In this article, FDFE was shown to be a valuable tool especially for guided wave mode selection in waveguides. It has the following advantages when compared to its time domain counterpart. They are:

1. It is computationally inexpensive;

2. Structural response of the waveguide to a single incident guided wave mode can be obtained and hence mode conversions can be more accurately studied; 
3. Phase velocities of the modes are obtained, as opposed to group velocities as in the time domain simulations;

4. Guided wave mode selection for inspecting targeted defects can be efficiently performed.

Finally, even though standard finite elements are generally used to solve the problem in the frequency domain, it might be more convenient and also computationally expedient to use other approaches like spectral finite elements [15]. Efforts in this direction are underway.

\section{Acknowledgements}

This material is mainly based upon work supported by the Nuclear Energy Universities Program under Award number 00102946, the National Sci-

ence Foundation under Award number 1300562. Baiyang Ren and Cliff J Lissenden also acknowledge the support of Government under the agreement No. W911W6-11-2-0011.

\section{References}

[1] J. L. Rose, A baseline and vision of ultrasonic guided wave inspection potential, Journal of pressure vessel technology 124 (3) (2002) 273-282. 
[2] J. L. Rose, Ultrasonic waves in solid media, Cambridge university press, 1999.

[3] K. F. Graff, Wave motion in elastic solids, Courier Corporation, 1991.

[4] A. Adamou, R. Craster, Spectral methods for modelling guided waves in elastic media, The Journal of the Acoustical Society of America 116 (3) (2004) 1524-1535.

[5] F. Moser, L. J. Jacobs, J. Qu, Modeling elastic wave propagation in waveguides with the finite element method, Ndt \& E International 32 (4) (1999) 225-234.

[6] J. Mu, J. L. Rose, Guided wave propagation and mode differentiation in hollow cylinders with viscoelastic coatings, The Journal of the Acoustical Society of America 124 (2) (2008) 866-874.

[7] P. Puthillath, J. M. Galan, B. Ren, C. J. Lissenden, J. L. Rose, Ultrasonic guided wave propagation across waveguide transitions: Energy transfer and mode conversion, The Journal of the Acoustical Society of America 133 (5) (2013) 2624-2633.

[8] P. Rajagopal, M. Drozdz, E. A. Skelton, M. J. Lowe, R. V. Craster, 
On the use of absorbing layers to simulate the propagation of elastic waves in unbounded isotropic media using commercially available finite element packages, NDT \& E International 51 (2012) 30-40.

[9] M. B. Drozdz, Efficient finite element modelling of ultrasound waves in elastic media, Ph.D. thesis, Imperial College London (2008).

[10] L. Moreau, A. Velichko, P. Wilcox, Accurate finite element modelling of guided wave scattering from irregular defects, Ndt \& E International 45 (1) (2012) 46-54.

[11] V. K. Chillara, C. J. Lissenden, Guided wave mode conversions across waveguide transitions: A study using frequency domain finite element method, in: 40TH ANNUAL REVIEW OF PROGRESS IN QUANTITATIVE NONDESTRUCTIVE EVALUATION: Incorporating the 10th International Conference on Barkhausen Noise and Micromagnetic Testing, Vol. 1581, AIP Publishing, 2014, pp. 308-315.

[12] M. H. Sadd, Elasticity: theory, applications, and numerics, Academic Press, 2009.

[13] C. Multiphysics, Comsol multiphysics user guide (version 4.3 a), COMSOL, AB. 
[14] B. Ren, C. J. Lissenden, Adhesive defect detection in composite adhesive joints using phased array transducers, in: 41ST ANNUAL REVIEW OF PROGRESS IN QUANTITATIVE NONDESTRUCTIVE EVALUATION: Volume 34, Vol. 1650, AIP Publishing, 2015, pp. 640-648.

[15] S. Gopalakrishnan, A. Chakraborty, D. R. Mahapatra, Spectral finite element method: wave propagation, diagnostics and control in anisotropic and inhomogeneous structures, Springer Science \& Business Media, 2007. 


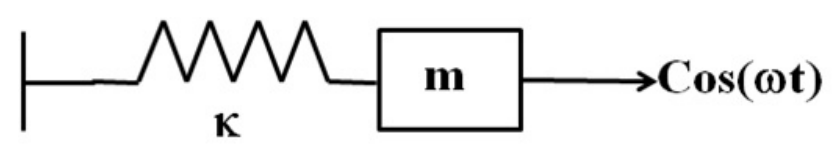

Figure 1: Schematic of spring-mass system

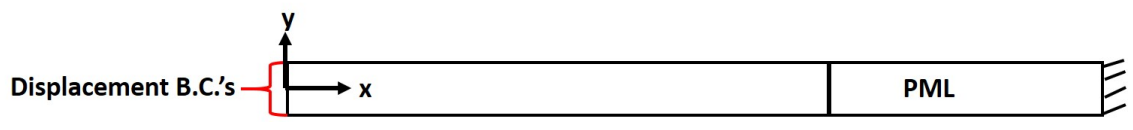

Figure 2: Schematic of the model used for the simulations 


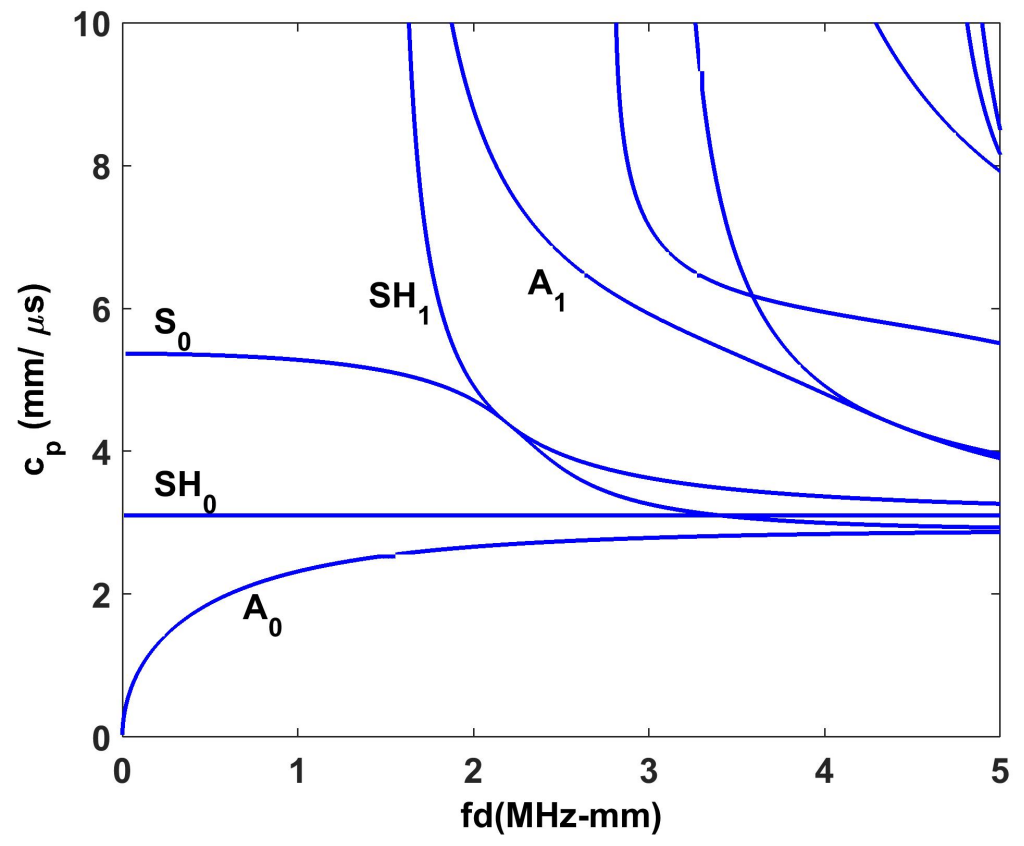

Figure 3: Dispersion curves for an aluminum plate 


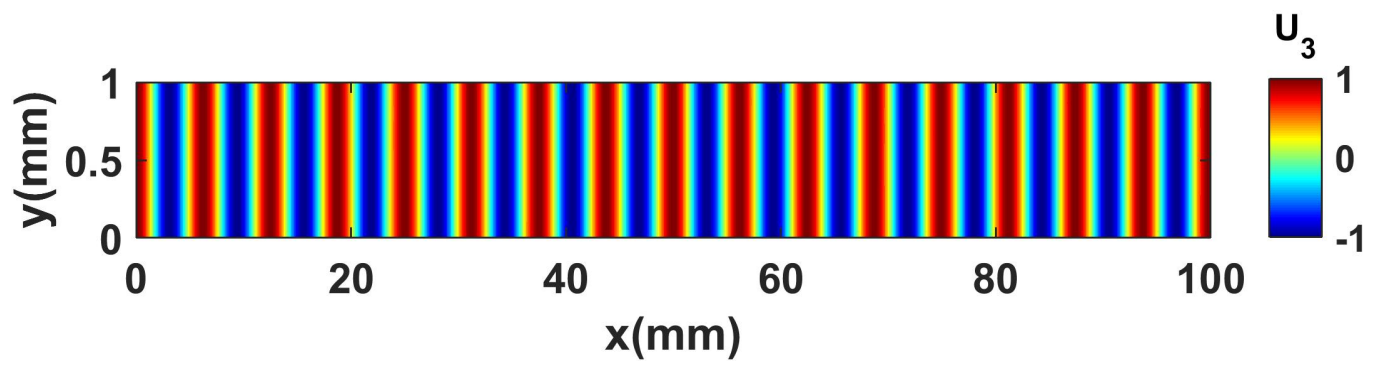

(a)

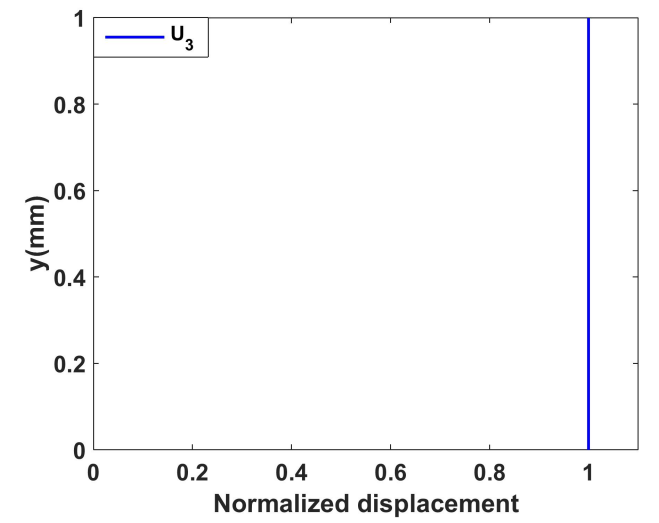

(b)

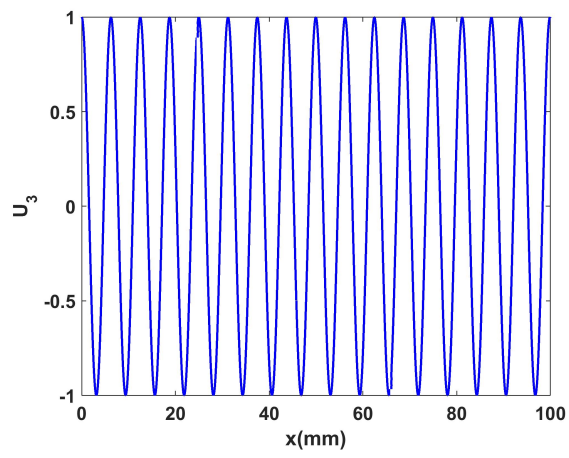

(c)

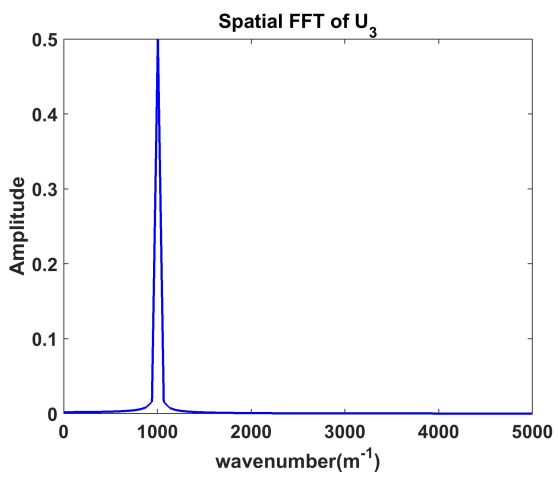

(d)

Figure 4: Symmetric $\mathrm{U}_{3}$ excitation at $0.5 \mathrm{MHz}$; (a) displacement field, (b) wavestructure, (c) displacement vs $x,(d)$ spatial Fourier transform of $\mathrm{U}_{3}$ 


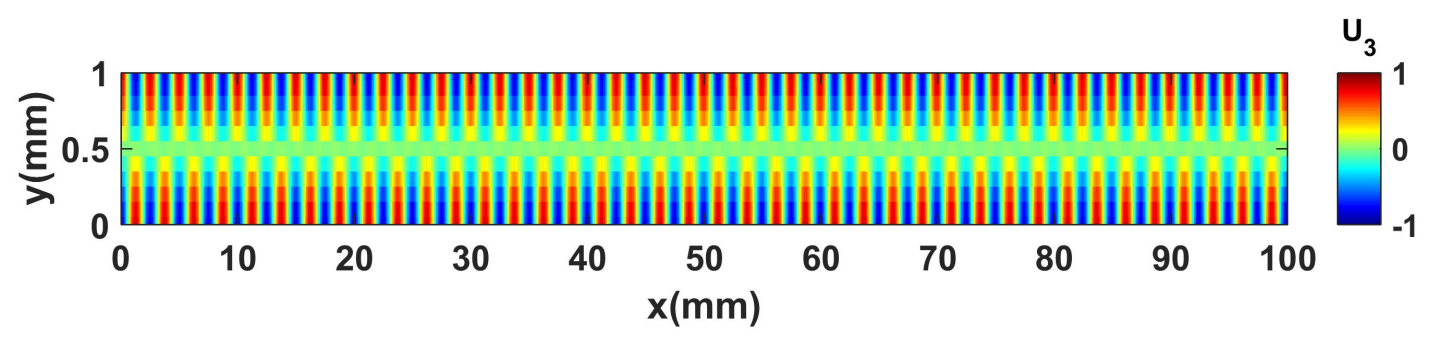

(a)

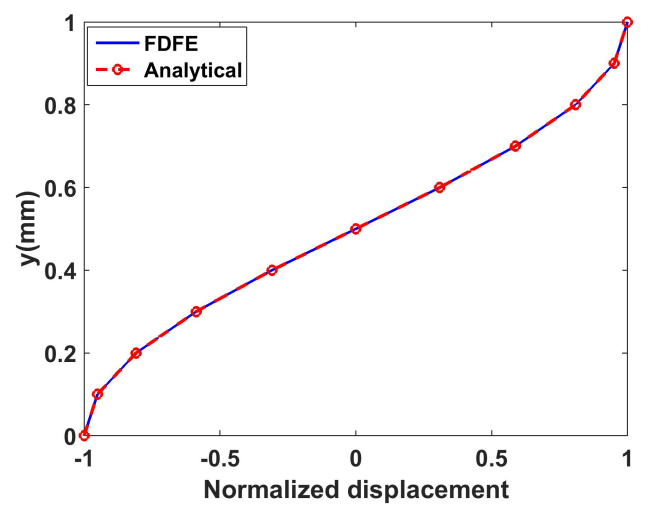

(b)

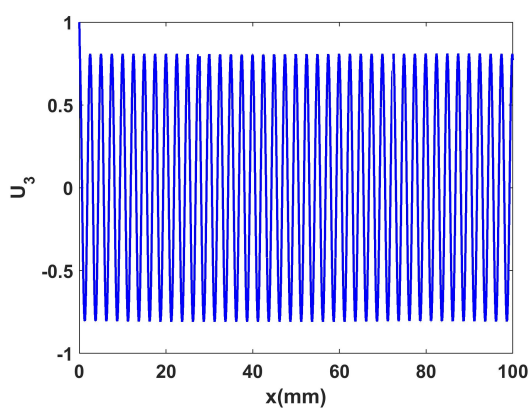

(c)

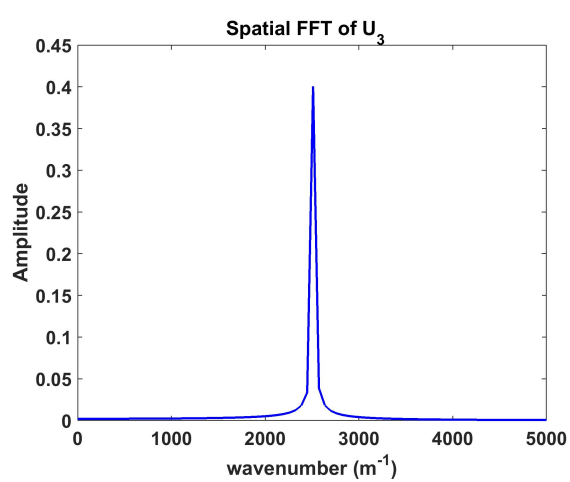

(d)

Figure 5: Antisymmetric $\mathrm{U}_{3}$ excitation at $2 \mathrm{MHz}$; (a) displacement field, (b) wavestructure, (c) displacement vs $x$, (d) spatial Fourier transform of $\mathrm{U}_{3}$ 


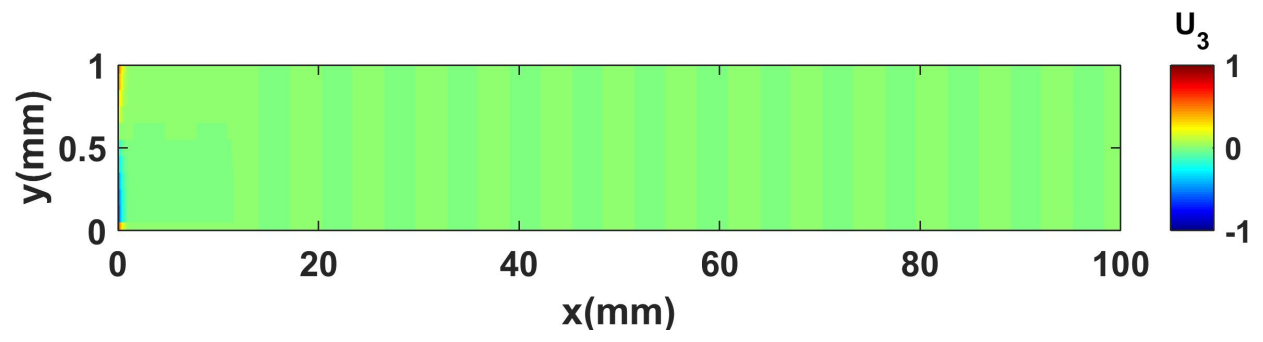

(a)

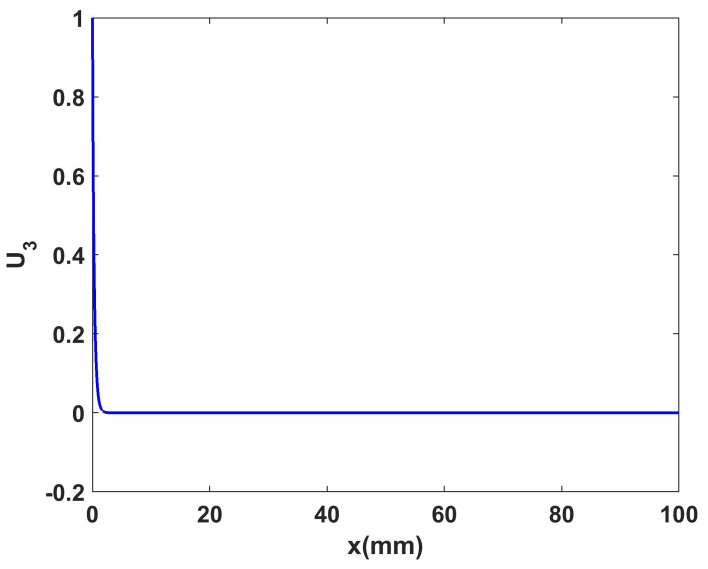

(b)

Figure 6: Antisymmetric $\mathrm{U}_{3}$ excitation at $0.5 \mathrm{MHz}$; (a) displacement field, (b) displacement vs $x$ 


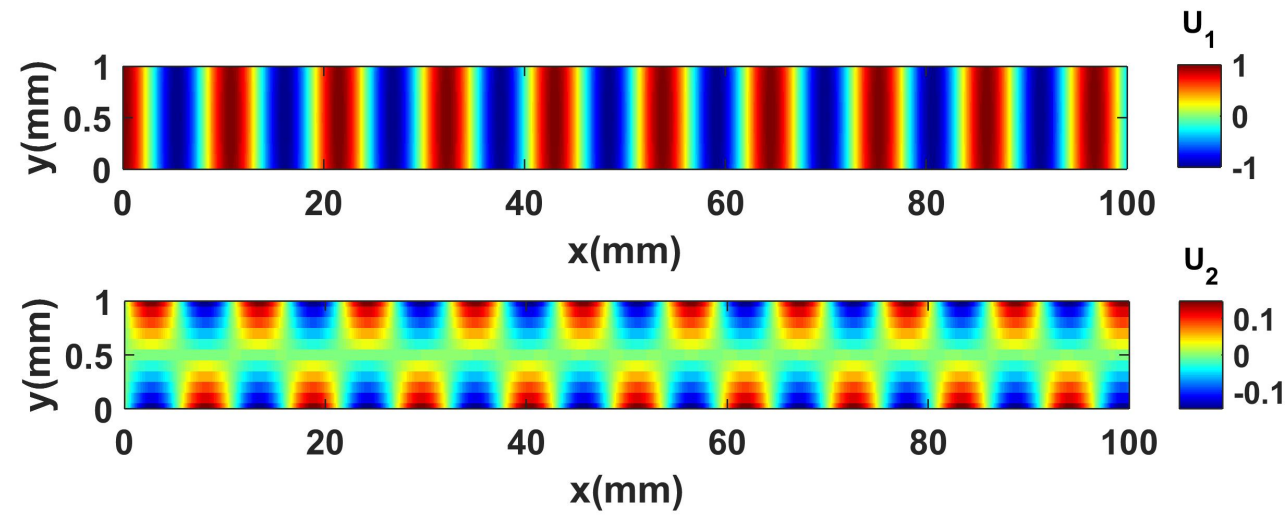

(a)

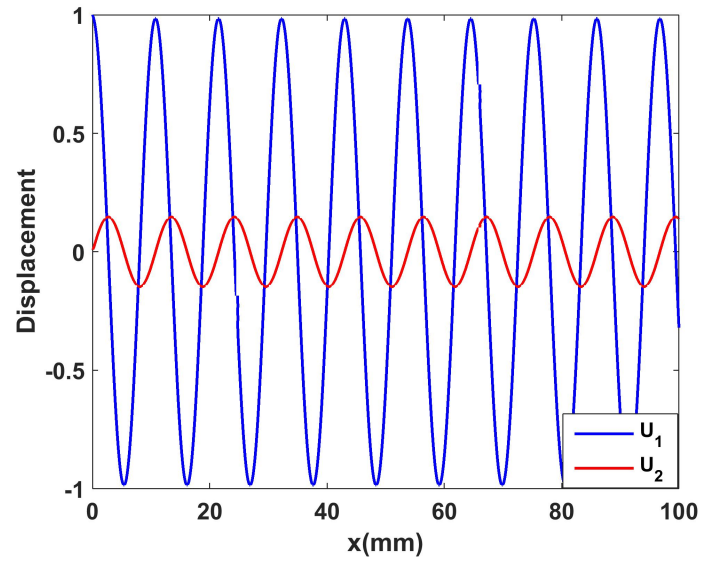

(b)

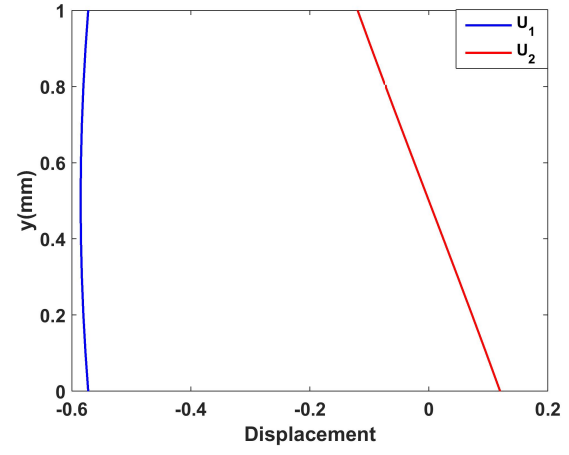

(c)

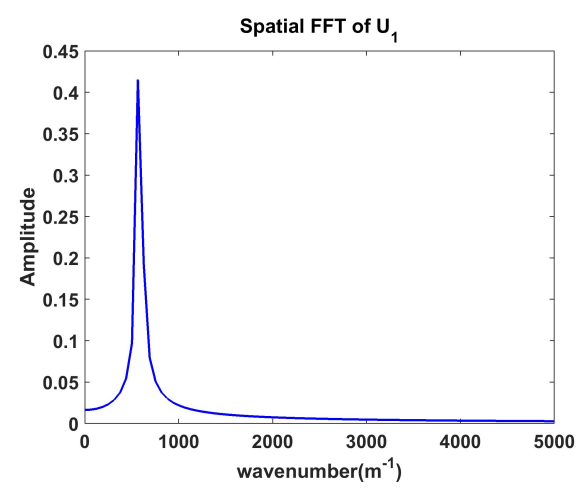

(d)

Figure 7: Symmetric $\mathrm{U}_{1}$ excitation at $0.5 \mathrm{MHz}$; (a) displacement field, (b) displacement vs $x,(c)$ Wavestructure (d) spatial Fourier transform of $\mathrm{U}_{1}$ along $x$. 


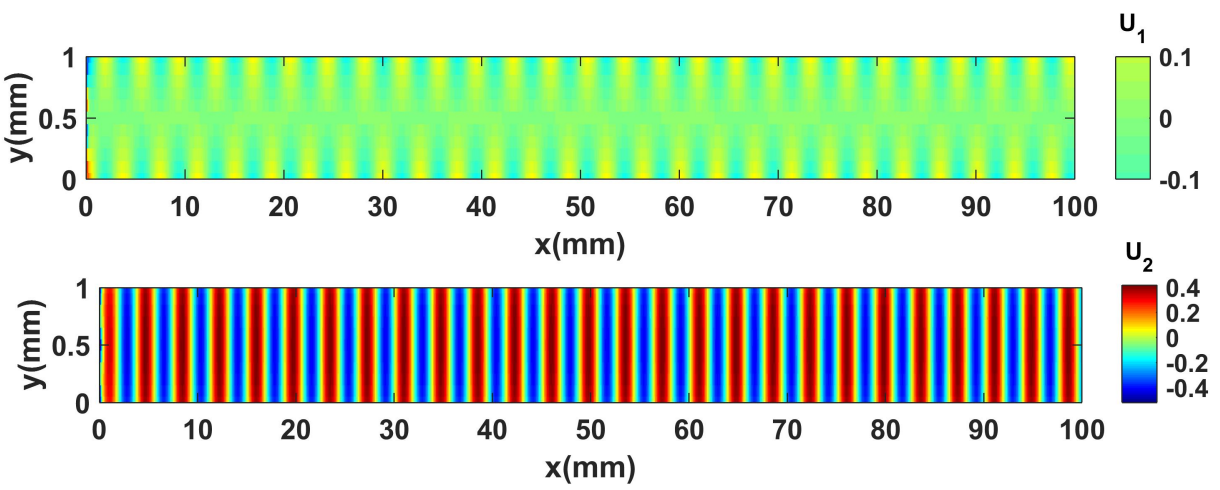

(a)

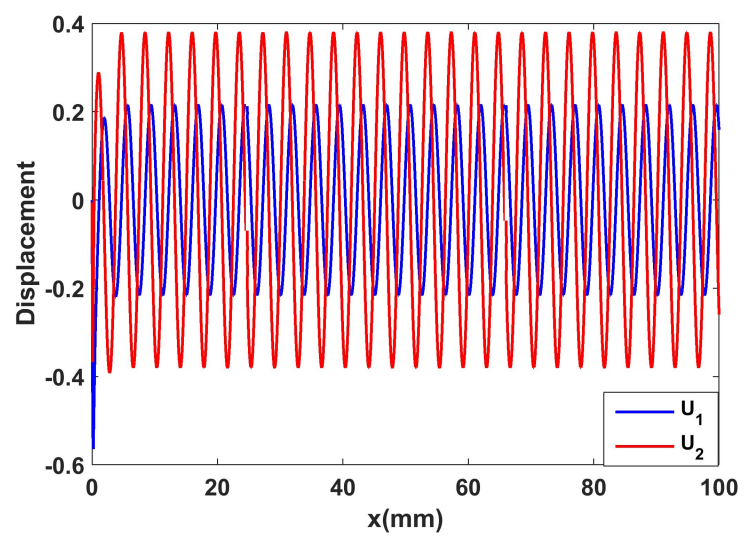

(b)

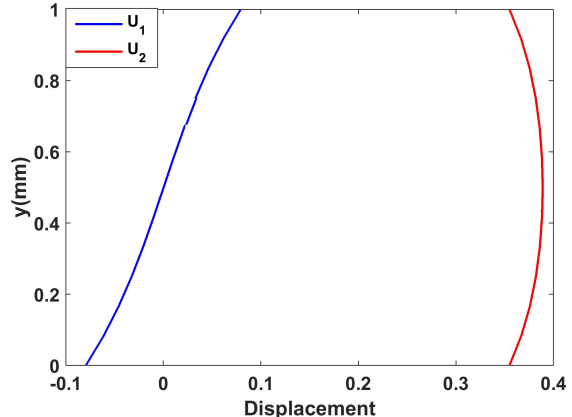

(c)

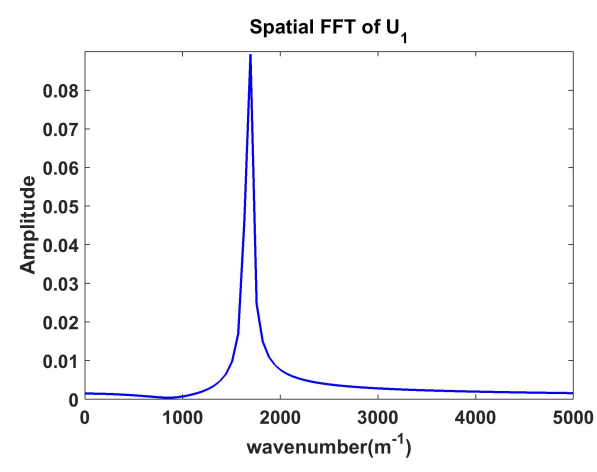

(d)

Figure 8: Antisymmetric $\mathrm{U}_{1}$ excitation at $0.5 \mathrm{MHz}$; (a) displacement field, (b) displacement vs $x$, (b)Wavestructure (d) spatial Fourier transform of $\mathrm{U}_{1}$ along $x$ 


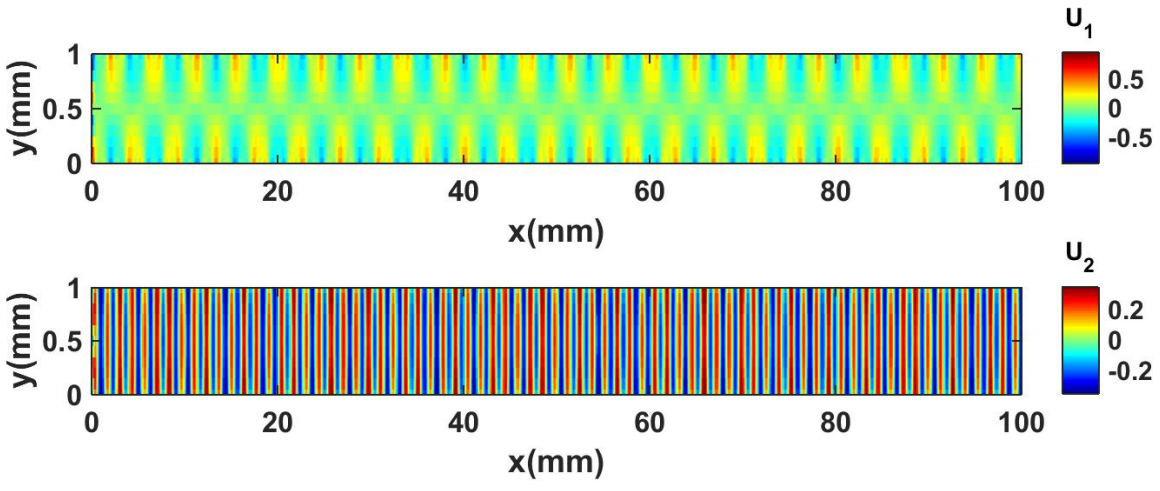

(a)

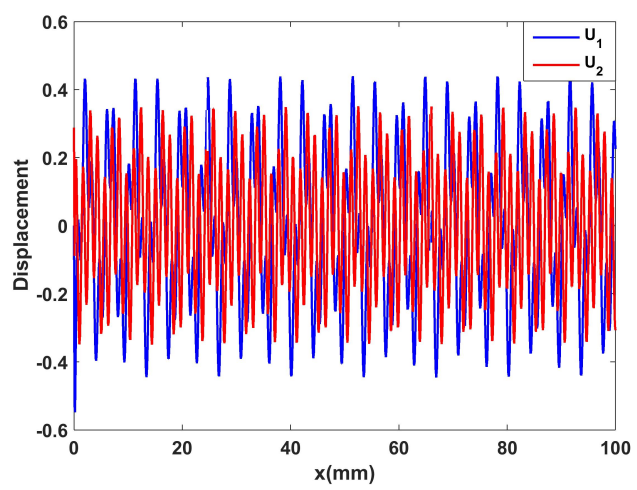

(b)

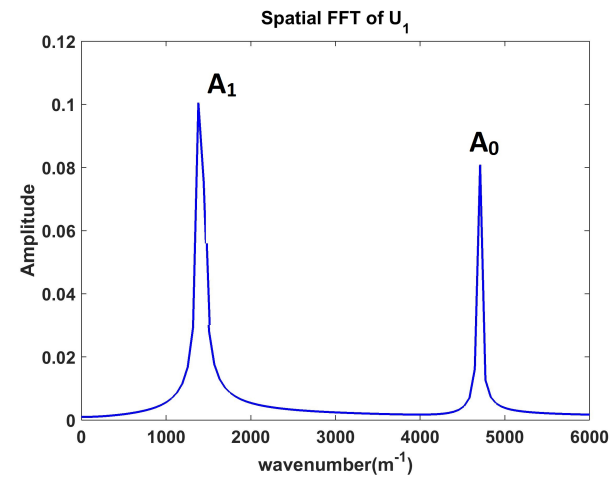

(c)

Figure 9: Antisymmetric $\mathrm{U}_{1}$ excitation at $0.5 \mathrm{MHz}$; (a) displacement field (b) displacement vs $x$, (c) spatial Fourier transform of $\mathrm{U}_{1}$ along $x$ 


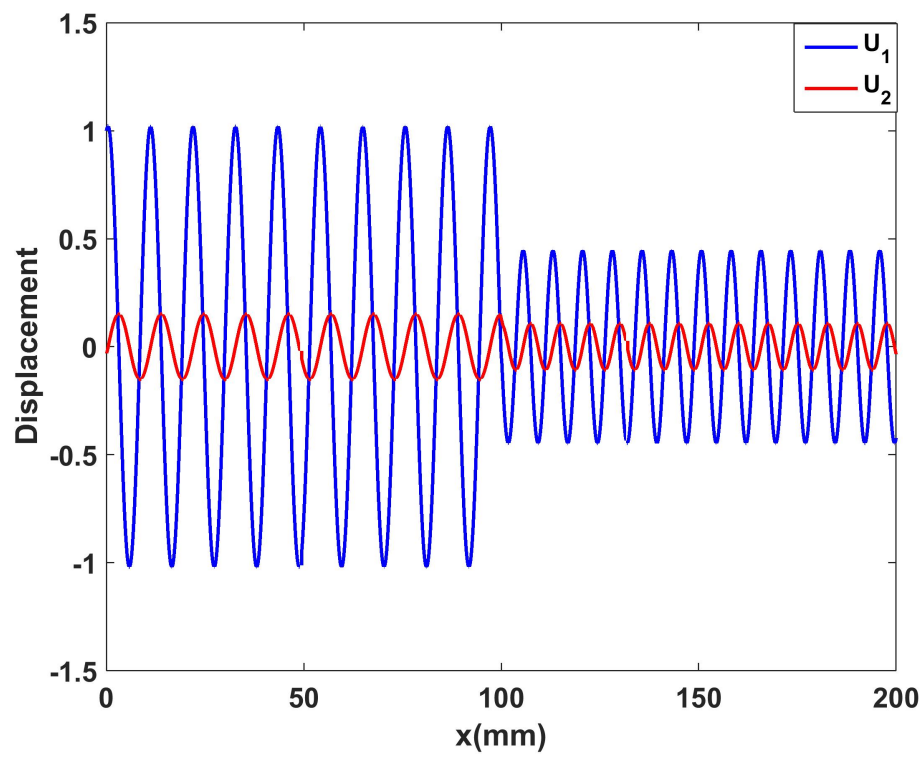

(a)

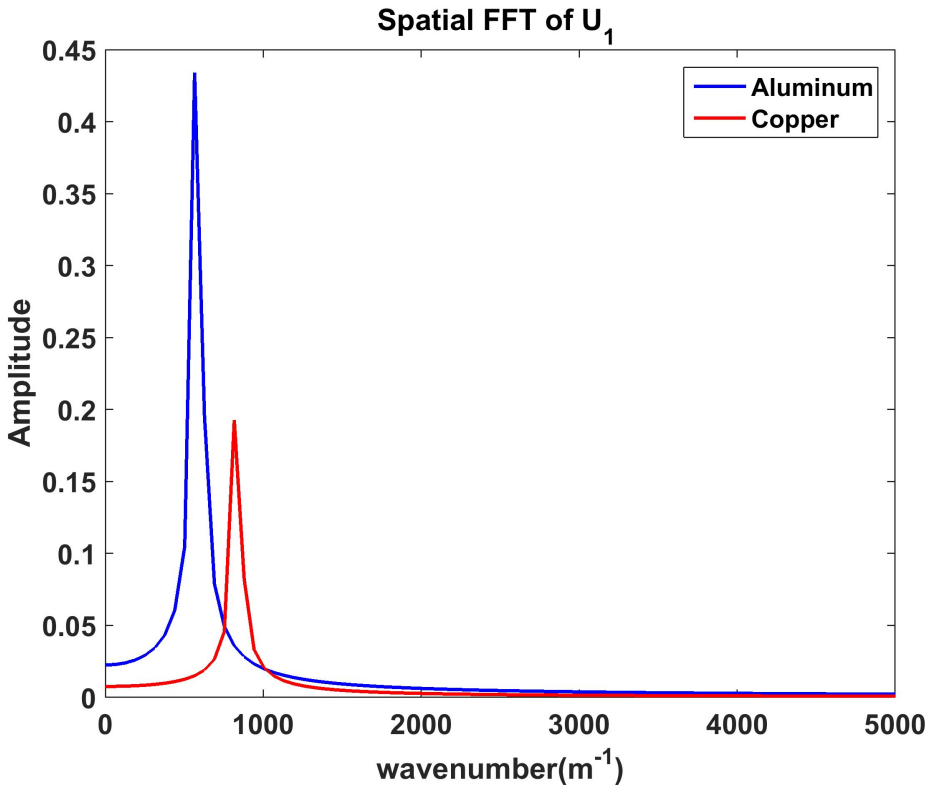

(b)

Figure 10: $\mathrm{U}_{1}=1$ BC; (a) Displacement with $x$ (b) Spatial FFT of $\mathrm{U}_{1}$ along $x$ 


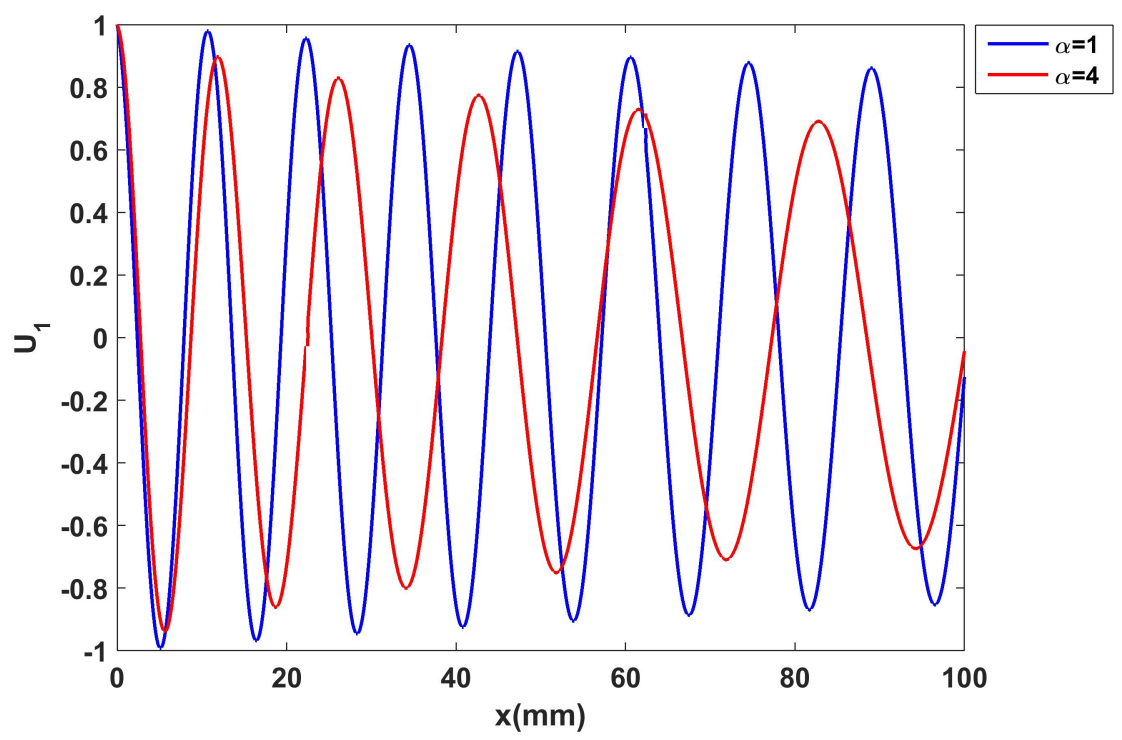

(a)

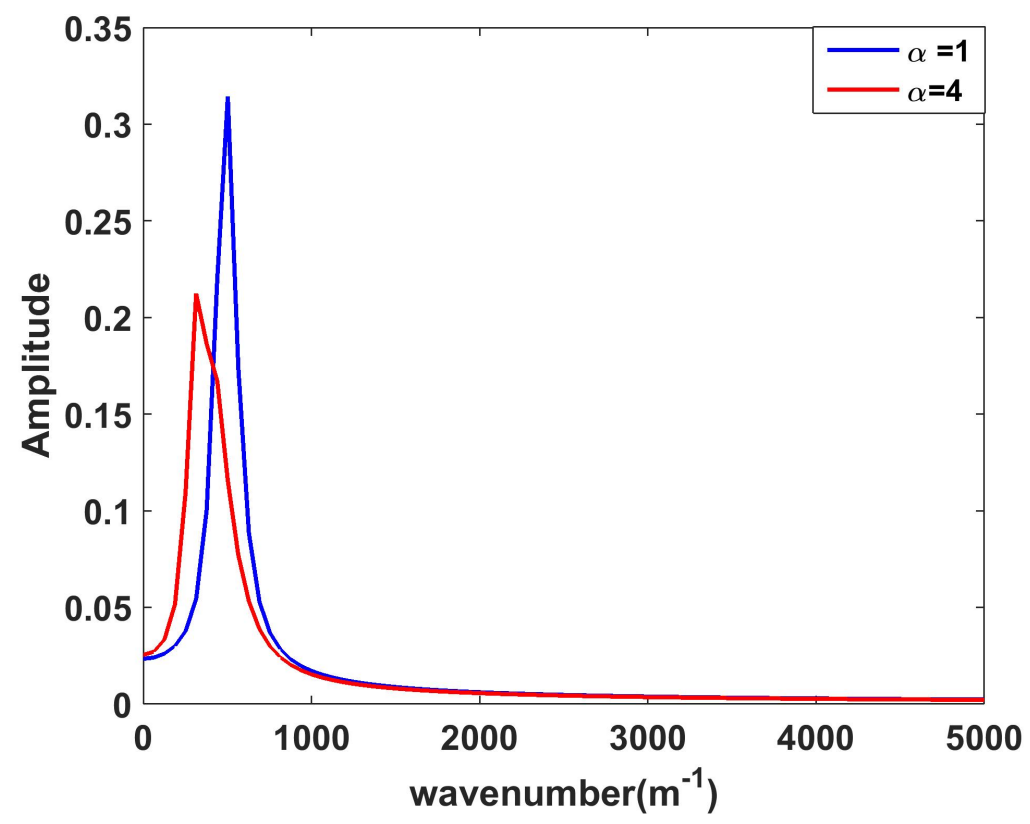

(b)

Figure 11: $\mathrm{U}_{1}=1$ BC; (a) Displacement with $x$ (b) Spatial FFT of $\mathrm{U}_{1}$ along $x$ for linearly graded plate. 


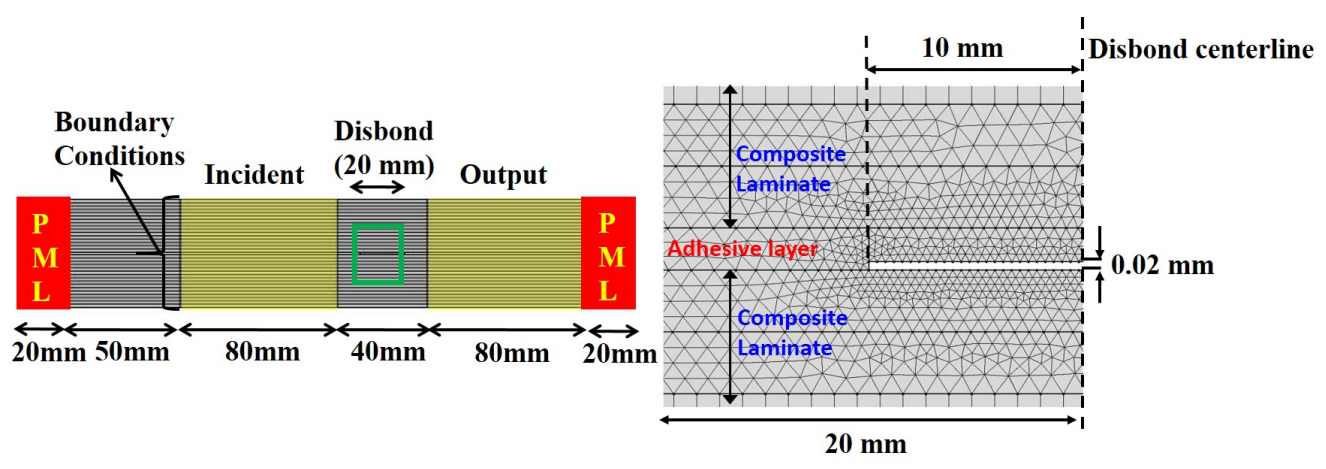

Figure 12: Schematic of the FDFE model used for simulation

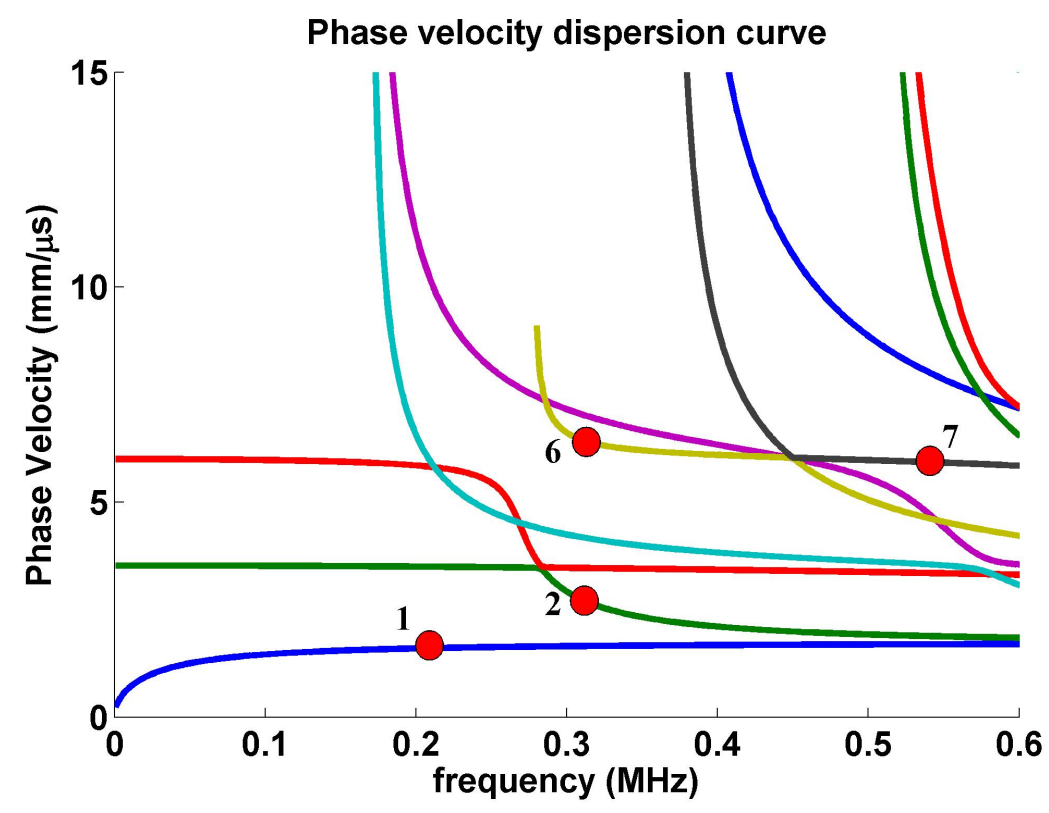

Figure 13: Dispersion curves for the CFRP plate. 


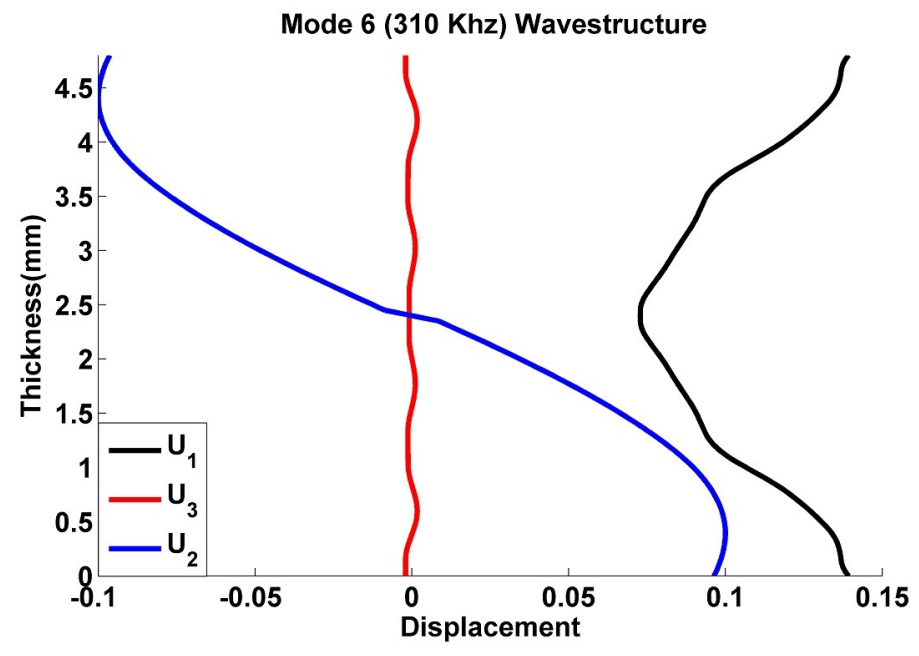

Figure 14: Wavestructure for Mode $6(310 \mathrm{kHz})$ 

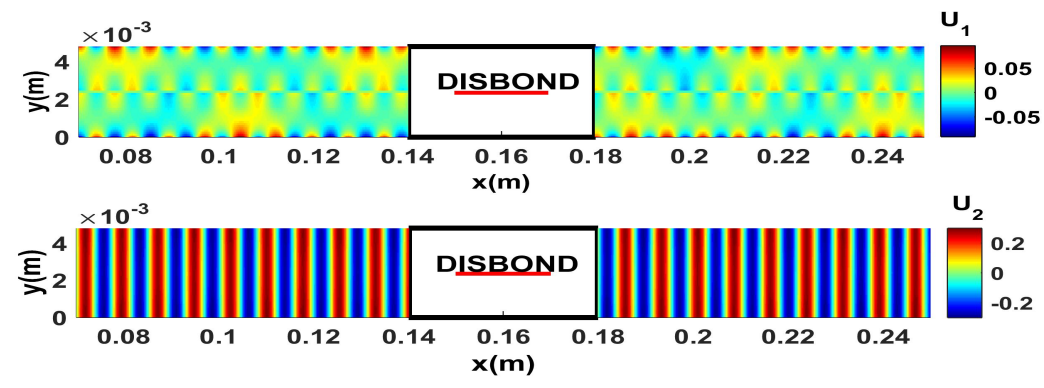

(a)
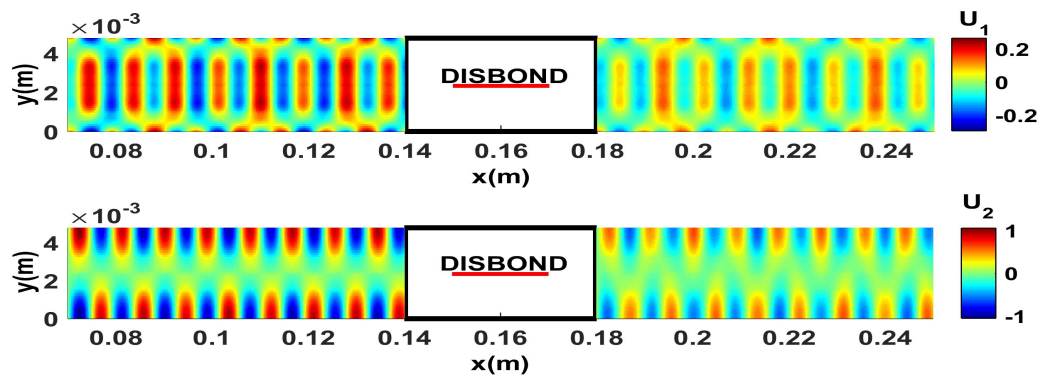

(b)
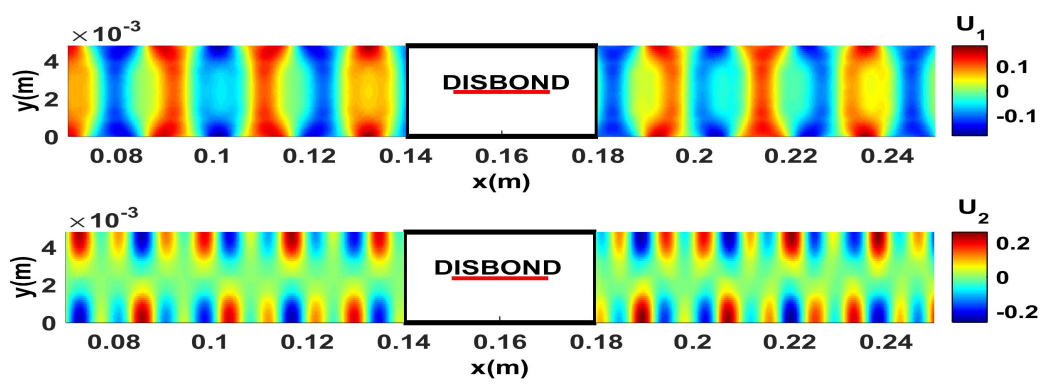

(c)
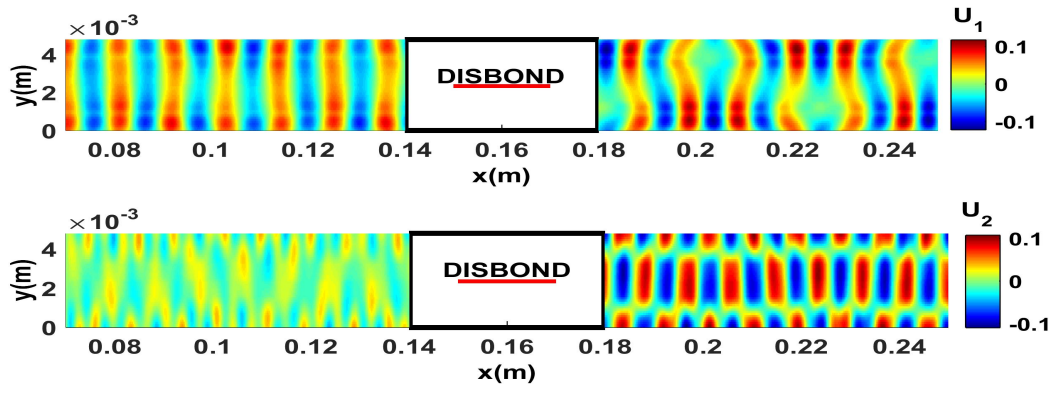

(d)

Figure 15: Displacement fields obtained from FDFE (a) Mode 1 (210 kHz) (b) Mode 2 $(310 \mathrm{kHz})(\mathrm{c})$ Mode $6(310 \mathrm{kHz})(\mathrm{d})$ Mode $7(540 \mathrm{kHz})$ 

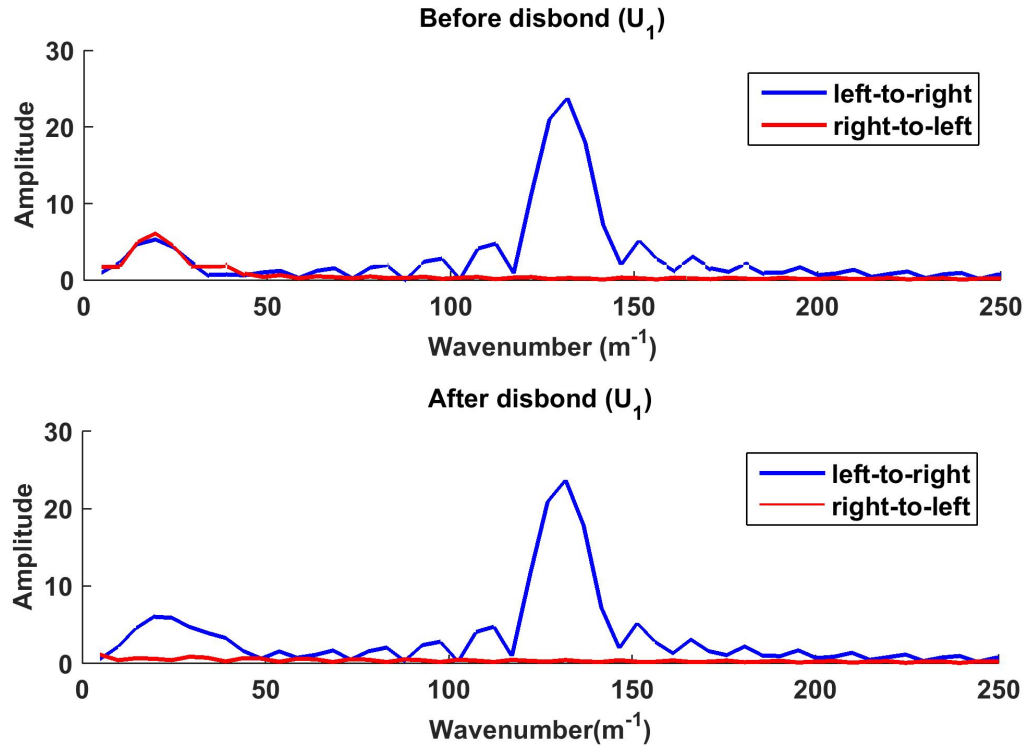

(a)
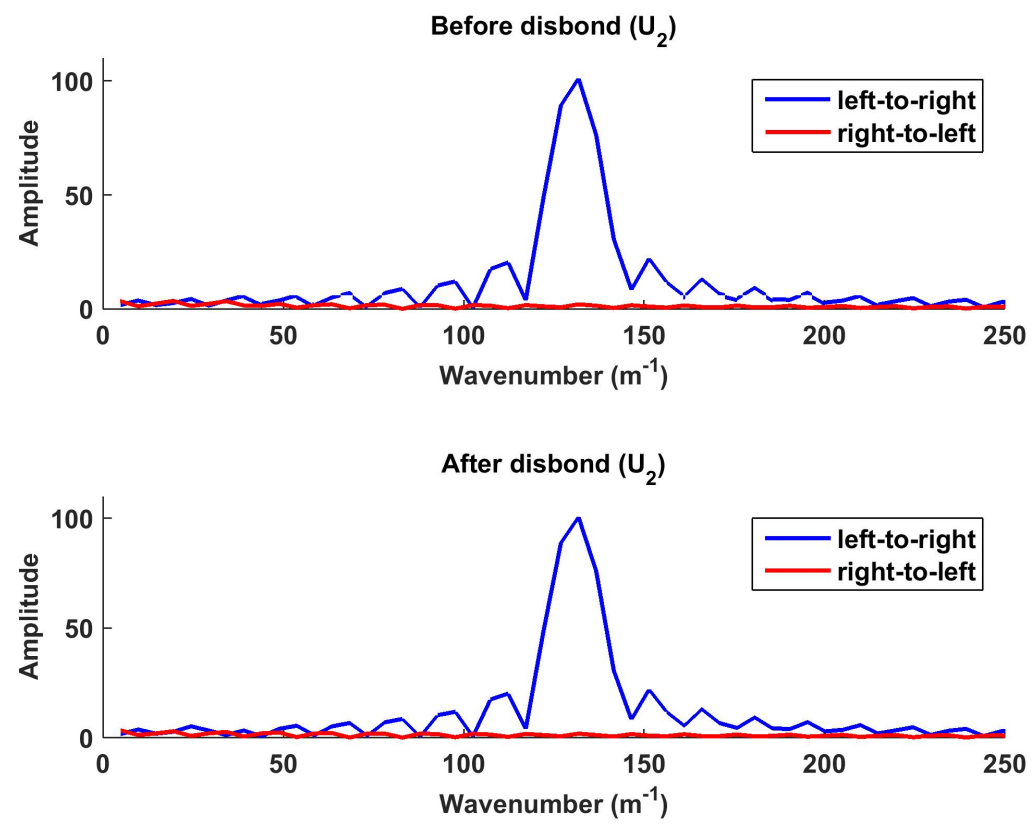

(b)

Figure 16: FFTs of the (a) $\mathrm{U}_{1}$ and (b) $\mathrm{U}_{2}$ displacement fields for the Mode $1(210 \mathrm{kHz})$ before and after the disbond. 

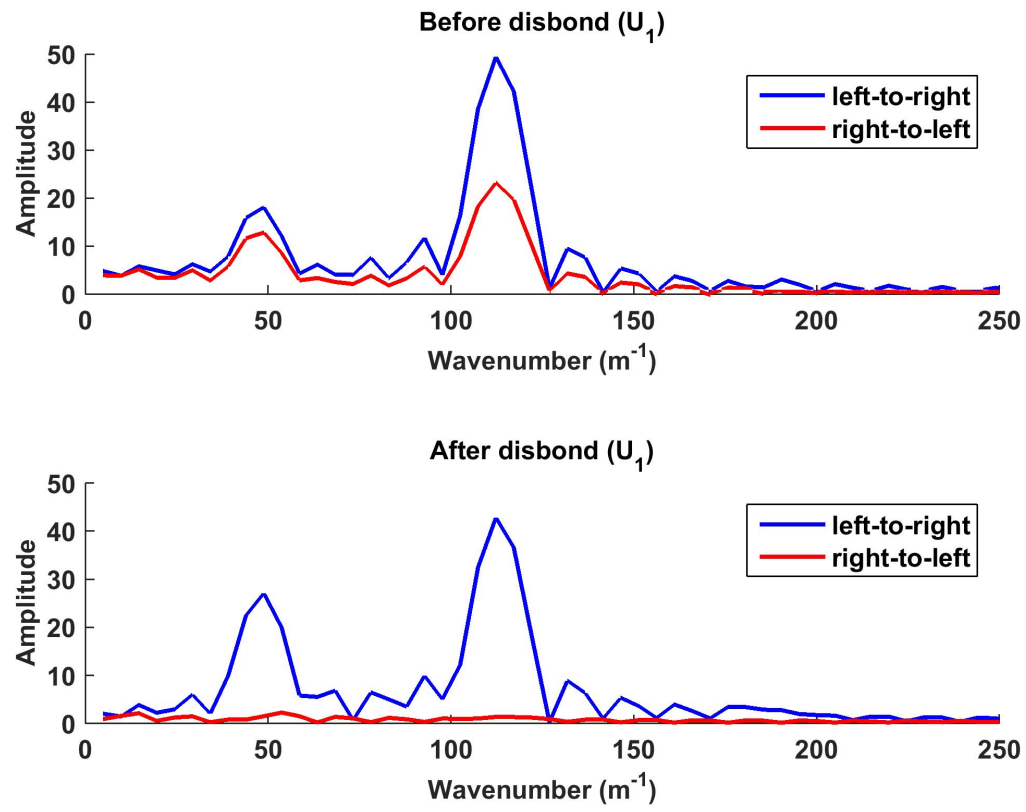

(a)
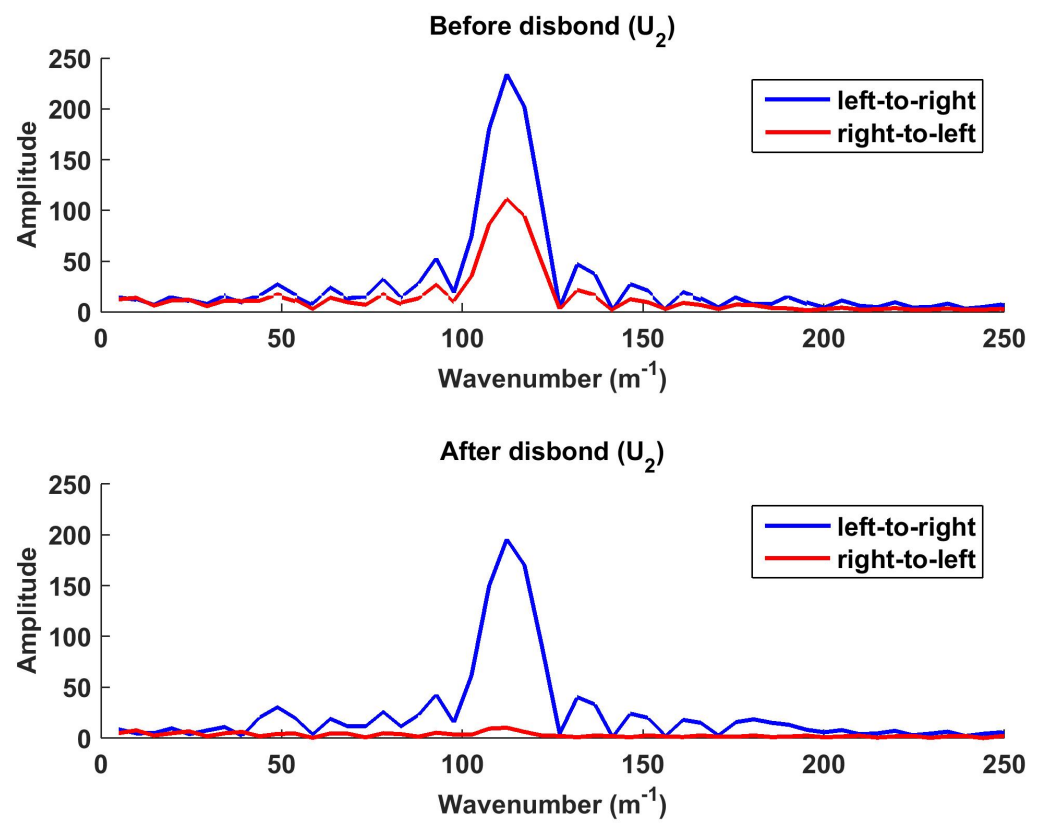

(b)

Figure 17: FFTs of the (a) $\mathrm{U}_{1}$ and (b) $\mathrm{U}_{2}$ displacement fields for the Mode $2(310 \mathrm{kHz})$ before and after the disbond. 


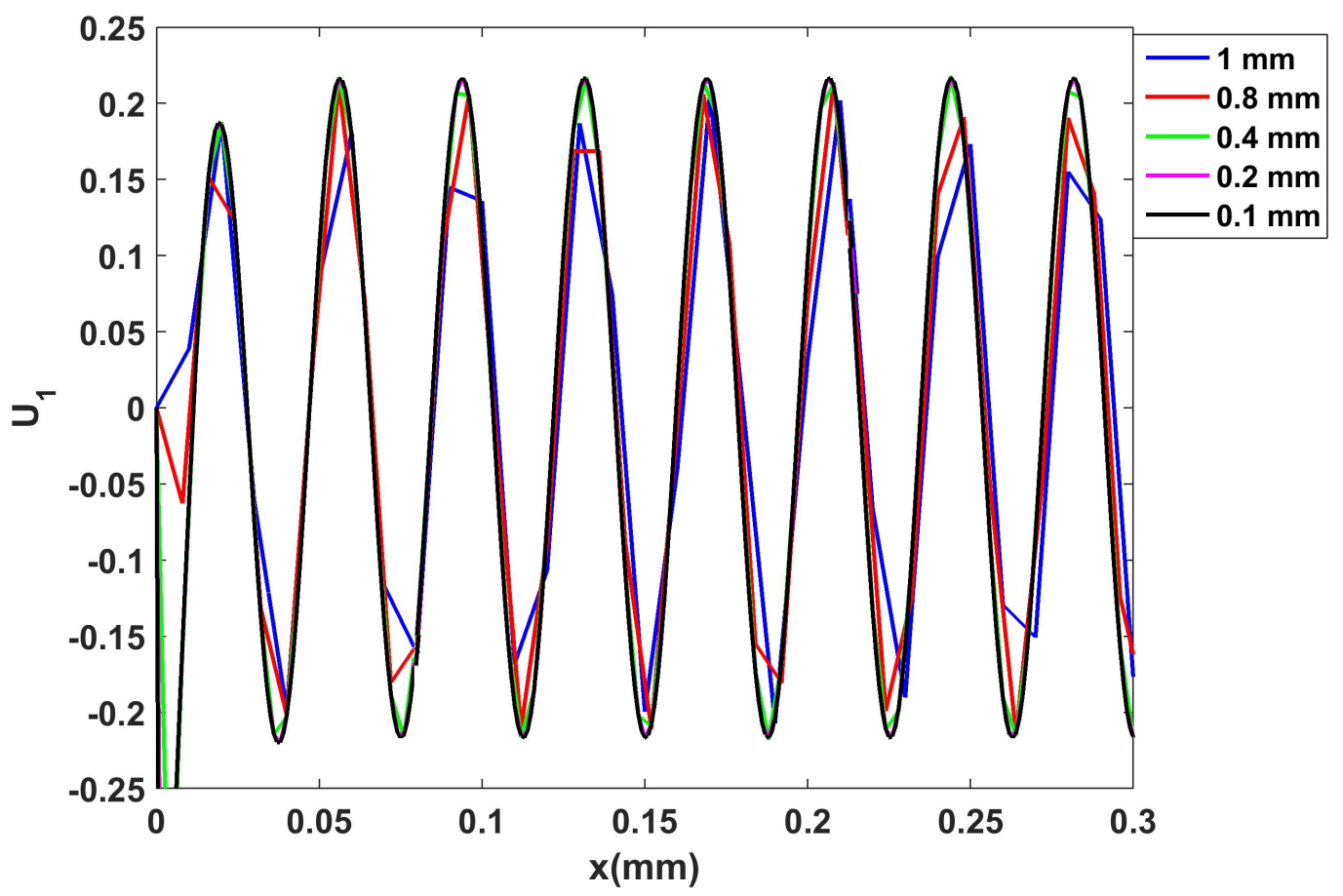

Figure 18: $\mathrm{A}_{0}$ mode $(0.5 \mathrm{MHz})$ - Displacement $\left(\mathrm{U}_{1}\right)$ with $x$ for different discretization. 


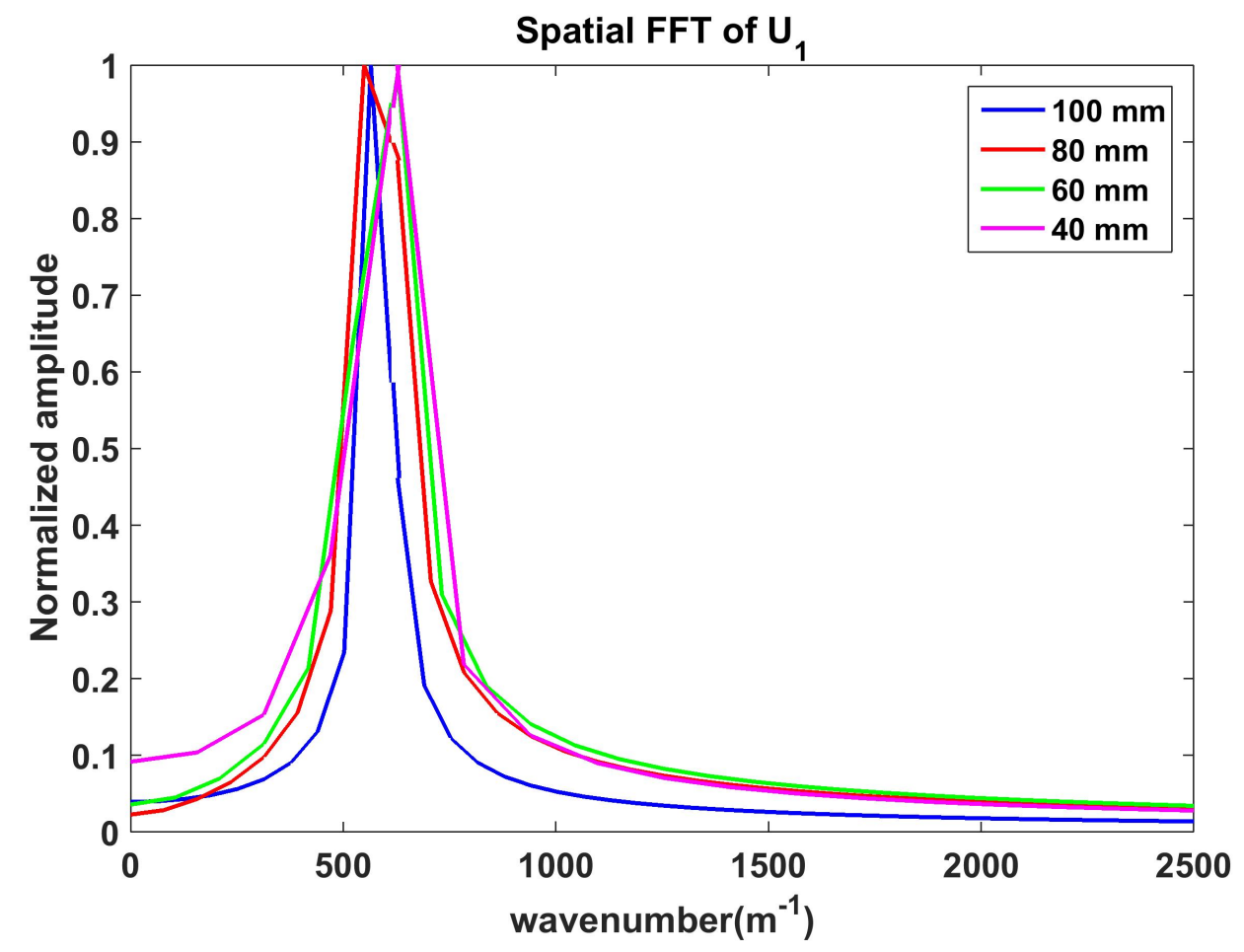

Figure 19: $\mathrm{A}_{0}$ mode $(0.5 \mathrm{MHz})$ - Spatial FFT of $\mathrm{U}_{1}$ for different waveguide lengths.

Table 1: Quasi-isotropic CFRP plate layup

\begin{tabular}{ccccc}
\hline $\begin{array}{l}\text { Laminate } \\
\text { thickness }\end{array}$ & $\begin{array}{c}\text { Adhesive } \\
\text { thickness }\end{array}$ & material (laminae) & Lay-up of laminae & Material (adhesive) \\
\hline \hline $2.35 \mathrm{~mm}$ & $0.1 \mathrm{~mm}$ & $\mathrm{AS} 4 / 8552$ & {$[0 / 45 / 90 /-45]_{s 2}$} & Epoxy
\end{tabular}


Table 2: Material Properties of AS4/8552 CFRP used for simulations

\begin{tabular}{ccccccc}
\hline Density $\left(\mathrm{kg} / \mathrm{m}^{3}\right)$ & $E_{1}(\mathrm{GPa})$ & $E_{2}(\mathrm{GPa})$ & $G_{12}(\mathrm{GPa})$ & $G_{23}(\mathrm{GPa})$ & $\nu_{12}$ & $\nu_{23}$ \\
\hline \hline 1580 & 135 & 9.5 & 4.9 & 4.9 & 0.3 & 0.45
\end{tabular}

Table 3: Material Properties of Epoxy used for simulations

\begin{tabular}{ccc}
\hline Density $\left(\mathrm{kg} / \mathrm{m}^{3}\right)$ & $E(\mathrm{GPa})$ & $\nu$ \\
\hline \hline 1104 & 2.56 & 0.402
\end{tabular}

